\title{
Labor income dynamics and the insurance from taxes, transfers, and the family
}

\author{
Richard Blundell ${ }^{\mathrm{a}, \mathrm{c}, *}$, Michael Graber ${ }^{\mathrm{a}, \mathrm{c}, \mathrm{d}}$, Magne Mogstad ${ }^{\mathrm{b}, \mathrm{c}, \mathrm{d}}$ \\ a Department of Economics, University College London, United Kingdom \\ b Department of Economics, University of Chicago, United States \\ c Institute for Fiscal Studies, United Kingdom \\ d Research Department, Statistics Norway, Norway
}

\section{A R T I C L E I N F O}

\section{Article history:}

Received 15 January 2013

Received in revised form 18 April 2014

Accepted 22 April 2014

Available online $\mathrm{xxxx}$

\section{JEL classification:}

C33

D3

D91

J31

\section{Keywords:}

Income dynamics

Insurance

Life cycle inequality

\begin{abstract}
A B S T R A C T
What do labor income dynamics look like over the life-cycle? What is the relative importance of persistent shocks, transitory shocks and heterogeneous profiles? To what extent do taxes, transfers and the family attenuate these various factors in the evolution of life-cycle inequality? In this paper, we use rich Norwegian population panel data to answer these important questions. We let individuals with different education levels have a separate income process; and within each skill group, we allow for non-stationarity in age and time, heterogeneous experience profiles, and shocks of varying persistence. We find that the income processes differ systematically by age, skill level and their interaction. To accurately describe labor income dynamics over the life-cycle, it is necessary to allow for heterogeneity by education levels and account for non-stationarity in age and time. Our findings suggest that the redistributive nature of the Norwegian tax-transfer system plays a key role in attenuating the magnitude and persistence of income shocks, especially among the low skilled. By comparison, spouse's income matters less for the dynamics of inequality over the life-cycle.
\end{abstract}

(C) 2014 The Authors. Published by Elsevier B.V. This is an open access article under the CC BY license

(http://creativecommons.org/licenses/by/3.0/).

\section{Introduction}

The aim of this paper is to examine the dynamics of labor income over the working life and to explore the impact of two mechanisms of attenuation or insurance to labor income shocks. The first is the tax and transfer system; the second is spouse's income. We focus on three dimensions of inequality: individual market income, individual disposable income, and family disposable income; and explore the relationship between them over the life-cycle. Our objective is to provide a detailed picture of the dynamics of inequality over the life-cycle, following individuals from many different birth cohorts across their working lifespan. By linking up individuals with other family members, we are able to examine the impact of spouse's income and the role of the tax-transfer system as mechanisms to smooth shocks to individual market income.

There are a number of key questions addressed. What do labor income dynamics look like over the life-cycle? What is the relative importance of persistent shocks, transitory shocks and heterogeneous profiles? To what

\footnotetext{
* Corresponding author at: Department of Economics, University College London, Gower Street, London WC1E 6BT, United Kingdom.

E-mail addresses: r.blundell@ucl.ac.uk (R. Blundell), michael.graber.09@ucl.ac.uk (M. Graber), magne.mogstad@gmail.com (M. Mogstad).
}

extent does the tax and transfer system attenuate these various factors in the evolution of life-cycle inequality? What happens when we add in income sources of spouses? Answering these questions has proved to be quite difficult. One problem that is often argued to hinder analysis is data availability. While the ideal data set is a long panel of individuals, this is somewhat a rare event and can be plagued by problems such as attrition and small sample sizes. An important exception is the case where countries have available administrative data sources. The advantages of such data sets are the accuracy of the income information provided, the large sample size, and the lack of attrition, other than what is due to migration and death.

To investigate the above questions, we exploit a unique source of population panel data containing records for every Norwegian from 1967 to 2006. Norway provides an ideal context for this study. It satisfies the requirement for a large and detailed data set that follows individuals and their family members over long periods of their working career. It also has a well developed tax-transfer system, and our data provides us with a measure of income pre and post the payment of taxes and the receipt of transfers. To understand the role of taxes, transfers and the family in attenuating shocks to labor income requires a model that allows for key aspects in the evolution of labor income over the life-cycle. The extensive literature on the panel data modeling of labor income dynamics points to three ingredients of potential significance: shocks of varying persistence; age and time dependence 
in the variance of shocks; and heterogeneous age profiles. For example, our long panel data allows us to decompose income shocks at every age into two components: one is mean-reverting over short periods (we label 'transitory' shocks), while the other could either be permanent or persistent and mean-reverting over long periods (we label 'permanent' shocks). By way of comparison, the usual random walk specification would restrict the permanent shocks to be truly permanent rather than merely persistent. Additionally, by following many different birth cohorts across their working lifespan we are able to allow a flexible structure for time effects in deriving our life-cycle profiles. ${ }^{1}$ The size and detailed nature of the data we are using allow us to explore the importance of these three ingredients for labor income dynamics.

Our key findings on the labor income dynamics of males are threefold. First, the magnitude of permanent and transitory shocks varies systematically over the life-cycle. Indeed, we may strongly reject the hypothesis of age-independent variance of shocks. Second, there is essential heterogeneity in the variances of permanent shocks across skill groups. For low skilled, the magnitude of permanent shocks is monotically increasing in age. For example, a permanent shock of one standard deviation implies a $35 \%$ change in individual market income for a low skilled 30 year old; the corresponding number for a low skilled 55 year old is $50 \%$. High skilled, on the other hand, experience large permanent shocks early in life; these shocks decrease in magnitude until age 35, after which they are relatively small and fairly stable. Third, the variance of transitory shocks exhibits a decreasing profile over the life-cycle. While this findings holds for all skill groups, high skilled tend to experience relatively large transitory shocks early in life.

The evidence of heterogeneity in the dynamics of labor income by age, skill level, and their interaction motivates and guides our analysis of the insurance from taxes, transfers and the family. We find that the tax-transfer system reduces both the level and persistence of shocks to labor income. In particular, taxes and transfers lead to a remarkable flattening of the age profiles in the variances of permanent and transitory shocks for the low skilled. At age 55 , for example, a permanent shock of one standard deviation implies a 50\% change in annual market income for a low skilled; the corresponding number for annual disposable income is only $31 \%$. After taking taxes and transfers into account, spouse's income matters little for the dynamics of inequality over the life-cycle.

Taken together, our results suggest that a progressive tax-transfer system could be an important insurance mechanism to labor income shocks, especially for low skilled. These results may have implications for both policy and a large and growing literature on consumption inequality and the overall ability of families to insure labor income shocks (see e.g. Blundell et al., 2012). Economic theory predicts that consumption responds strongly to highly persistent or permanent shocks, and empirical evidence suggests little if any self-insurance in response to permanent shocks among individuals with no college education (see e.g. Blundell et al., 2008). Our study points to the importance of understanding the nature of risk that families face over the life-cycle, and the extent to which taxes and transfers crowd out or add to the insurance available in financial markets, the family or other informal mechanisms.

Our paper also contributes to the literature on modeling of labor income dynamics. Identification of credible income processes is key for answering a number of important economic questions, including lifecycle consumption and portfolio behavior (see e.g. Gourinchas and Parker, 2002), the sources of inequality (see e.g. Huggett et al., 2011), and the welfare costs of business cycles (see e.g. Storesletten et al., 2001, 2004). The conclusions reached about these questions likely

\footnotetext{
1 See, for example, the recent review by Meghir and Pistaferri (2011), and the extensive list of studies referenced therein. These studies build and extend on the original papers by MaCurdy (1982) who developed the permanent transitory framework, Moffitt and Gottschalk (2012) who show the potential importance of time dependence in the variance components, and Lillard and Weiss (1979) and Guvenen (2009) who established a role of heterogeneous profiles.
}

depend on the specification of the labor income process used to calibrate the models. The relatively small scale of the available U.S. panel surveys has forced researchers to rely on simple models that impose economically implausible restrictions (see the discussions in Baker and Solon, 2003; Meghir and Pistaferri, 2011). Using rich Canadian data from 1976 to 1992, Baker and Solon (2003) reject several of these restrictions, including no life-cycle variation in the variance of transitory shocks. ${ }^{2}$ DeBacker et al. (2013) use a large panel of tax returns to study income dynamics in the U.S. over the period 1987-2009. ${ }^{3}$ Their estimates point to the importance of allowing for time dependence in the variance components of income.

Our study complements these studies by bringing new evidence on several issues pertinent to modeling of income processes. One key finding is that allowing for both age and time dependence in the variance components is essential to accurately describe labor income dynamics. In particular, when restricting the variances of the error components to be constant across the life-cycle, we miss the large permanent shocks that occur late (early) in life for the low (high) skilled. Another key finding is that allowing for heterogeneity by education levels is necessary to capture labor income dynamics of young and old workers. When we restrict the income processes at the variance level to be the same across skill groups, we find a U-shaped age profile in the variances of permanent shocks; however, this pattern is at odds with the age profiles of both high and low skilled. ${ }^{4}$ By way of comparison, the dynamics of income over the life-cycle change little when restricting the transitory shocks to be uncorrelated over time or allowing for heterogeneous experience profiles within each skill group. Indeed, only for the high skilled, there is evidence of significant unobserved heterogeneity in the income growth rates. Accounting for this heterogeneity lowers the overall persistence of income shocks somewhat, but barely moves the age profiles in the variances of permanent and transitory shocks.

The remainder of the paper proceeds as follows. Section 2 presents our data and discusses institutional details. Section 3 describes our panel data specification for income dynamics and presents our findings on the labor income process of males. Section 4 explores the degree of insurance provided by taxes and transfers as well as the income of the spouse. Section 5 offers evidence on several issues pertinent to modeling of income processes. Section 6 concludes.

\section{Data and institutional details}

\subsection{Data and sample restrictions}

Our analysis employs several registry databases maintained by Statistics Norway that we can link through unique identifiers for each individual. This allows us to construct a rich longitudinal data set containing records for every Norwegian from 1967 to 2006. The variables captured in this data set include individual demographic information (including gender, date of birth, and marital status) and socioeconomic information (including years of education, market income, cash transfers). The data contains unique family identifiers that allow us to match spouses and parents to their children.

The coverage and reliability of Norwegian registry data are considered to be exceptional (Atkinson et al., 1995). Educational attainment is reported by the educational establishment directly to Statistics Norway, thereby minimizing any measurement error due to

\footnotetext{
${ }^{2}$ Like Moffitt and Gottschalk (2012) and Haider (2001), Baker and Solon (2003) go beyond earlier models by allowing for changes over calendar time in both the persistent and transitory components of income. However, Baker and Solon (2003) do not allow for lifecycle variation in the variance of permanent shocks. Ostrovsky (2010) extends the analysis in Baker and Solon (2003) to the period from 1985 to 2005. See also Ostrovsky (2012), who uses Canadian data to estimate a model which allows for separate correlation of spouse's permanent and transitory components.

${ }^{3}$ See also Kopczuk et al. (2010).

${ }^{4}$ Using PSID, Karahan and Ozkan (2013) also find a U-shaped age profile in the variances of permanent shocks.
} 
misreporting. More importantly, the Norwegian income data have several advantages over those available in many other countries. First, there is no attrition from the original sample because of the need to ask for permission from individuals to access their tax records. In Norway, these records are in the public domain. Second, our income data pertain to all individuals and all jobs, and not only to jobs covered by social security. Third, we have nearly career-long income histories for certain cohorts, and do not need to extrapolate the income profiles to ages not observed in the data. And fourth, there are no reporting or recollection errors; the data come from individual tax records with detailed information about the different sources of income.

We study income dynamics for the 1925-1964 annual birth cohorts during the period 1967-2006. The reason for this selection of cohorts is to ensure fairly long records on earnings for each individual. We restrict the sample to males, to minimize selection issues due to lower labor market participation rates for women in the early periods. In line with much of the previous literature, we exclude immigrants and selfemployed. We further refine the sample to be appropriate for our analysis of labor income dynamics. In each year, we select males who are between the ages of 25 and 60 . These individuals will likely have already completed most of their schooling and are too young to be eligible for early retirement schemes. In our baseline specification, we further restrict the sample to individuals with at least four subsequent observations with positive market income. This restriction gives us the largest possible sample, given that transitory shocks are assumed to follow a first-order moving average process.

Applying these restrictions provides us with a panel data set with 40 time periods and 934,704 individuals. We will refer to this as the baseline sample. On average, this sample consists of 23,368 individuals per birth cohort. Our model estimates age-specific variance components from age 26 to 58. By following many birth cohorts, we are able to allow a flexible structure for calendar time effects in deriving our lifecycle profiles. For the 1942-1946 cohorts, we observe income at every age. For the cohorts born earlier (1925-1941), we miss one or more income observations between the ages of 25 and 41. For the cohorts born later (1947-1964), income is no longer observed at some point after age 42. As a result, our age-specific estimates are based on an unbalanced panel of income. Appendix Fig. C.1 shows the sample size by age. The number of observations declines late (early) in the working lifespan because we are not observing the labor income of younger (older) cohorts at these ages. It is therefore reassuring that the mean and variance of income display similar shapes over the life cycle across cohorts.

The income variables that we consider are defined as follows. The first variable is individual market income, defined as the annual pretax earnings. ${ }^{5}$ The second variable is individual disposable income, incorporating annual earnings and cash transfers net of taxes. ${ }^{6}$ The third variable is family disposable income. Our measure of family disposable income pools the individual disposable income of the spouses (if the male has a spouse).

Throughout the analysis, we partition the baseline sample into three mutually exclusive groups according to educational levels. The reason is that previous studies point to heterogeneity in the dynamics of labor income by educational levels. Low skilled is defined as not having completed high school (32\% of the baseline sample), medium skilled includes individual with a high school degree (48\% of the baseline sample), and the high skilled consists of individuals who have attended college ( $20 \%$ of the baseline sample).

\footnotetext{
${ }^{5}$ Unfortunately, we are unable to measure hourly wages because we do not have data on working hours.

${ }^{6}$ Our measure of disposable income excludes income from financial assets and subtracts taxes on earnings and cash transfers. In every year, we compute taxes using a tax simulation program available at Statistics Norway. Due to data availability, our measure of cash transfers omits certain short-term and work-related benefits, including sickness pay and unemployment benefits.
}

\subsection{Institutional details and descriptive statistics}

Before turning to the estimation of the income processes, we describe a few important features of our data and the Norwegian setting.

We first consider the pattern of labor market participation over the life cycle. Appendix Fig. C.2 shows the population share of males with positive market income by age. We see that the labor market participation rate starts out at around $90 \%$ when individuals are young. The participation rate remains at this high level until individuals reach their 50s, at which point they start exiting the labor market at an increasing rate. In particular, low skilled individuals are relatively likely to exit the labor market before they can receive (early) retirement benefits.

Appendix Fig. C.3 shows the levels and growth rates in market income by the age at which individuals exit the labor market. We see that early exits from the labor market are associated with low and declining market income in the years prior to exit. Given the sample restriction of non-zero market income, our baseline sample will therefore be of higher quality toward the end of the lifecycle (especially among the low educated). This should put downward pressure on the magnitude of shocks late in life, and most likely give us a lower bound on the insurance from taxes and transfers at these ages.

Next, we consider how individual market income varies over the life-cycle in our baseline sample. Fig. 1 shows the age profiles in the different measures of income by education levels. Each market income profile displays the familiar concave shape documented and analyzed by Mincer (1974), but the college-educated workers experience more rapid market income growth early in the working lifespan. Fig. 2 shows the variance of log market income over the life-cycle according to education levels. In line with the prediction of the Mincer model, the variances of medium and high skilled have a U-shaped profile. ${ }^{7}$ Among low skilled, the variance of log market income is weakly increasing until they are in their mid 40s, after which it rises rapidly.

We then examine the extent to which the tax and transfer system affects the mean and the variance of log individual income over the lifecycle. Fig. 1 show how the progressive nature of the tax system dampens the income differentials between high skilled and low skilled after age $35 .{ }^{8}$ At the same time, low skilled are more likely to receive cash transfers while working (such as partial disability benefits), especially toward the end of the working lifespan. Fig. 2 shows how the tax-transfer system eliminates the increase over the life-cycle in the variance of log market income among the low educated. By comparison, taxes and transfers do less to the large income variance among the high skilled early in their careers.

Lastly, we consider how family disposable income varies over the life-cycle in our baseline sample. Fig. 1 compares the age profiles in log family disposable income and log individual disposable income. In the beginning of the working lifespan, relatively few males are married and individual disposable income is therefore quite similar to family disposable income. ${ }^{9}$ When the males are in their mid 30s, the vast majorities are married and the income of the spouse plays a more important role. At this point, about $80 \%$ of the spouses are participating in the labor market, thus contributing significantly to family income. ${ }^{10}$ Fig. 2 shows the life-cycle variation in the variance of log family disposable income. The family income measure displays quite similar variance over the working lifespan as compared to the measure of individual disposable income.

\footnotetext{
7 Using Census data from the U.S., Heckman et al. (2003) also find that the variance of log labor income over the life has a U-shaped pattern.

8 The tax system is progressive through deductions and surtaxes. Appendix Figs. B.1 and B. 2 show the tax rates on market income in different years.

9 Appendix Fig. C. 4 shows the share of the sample that is married by age and educational levels.

${ }^{10}$ Appendix Fig. C.5 shows the labor market participation rate of the spouses by age and educational levels.
} 
a) Low-Skilled

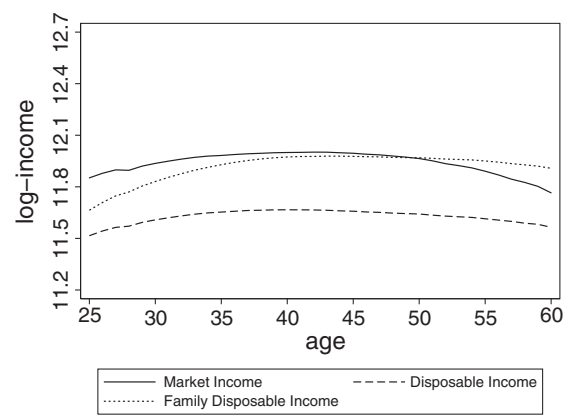

b) Medium-Skilled

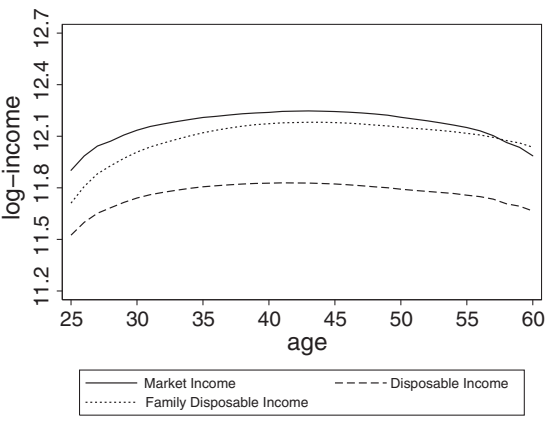

c) High-Skilled

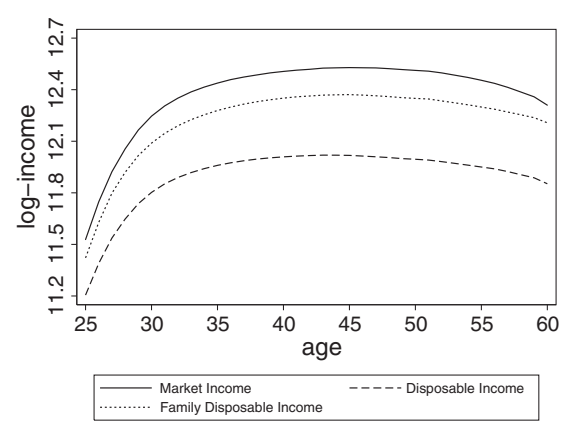

Fig. 1. Age profiles in the log of income.

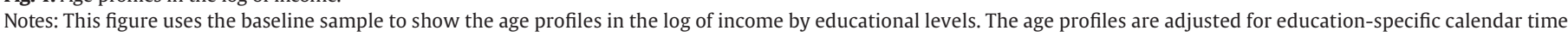

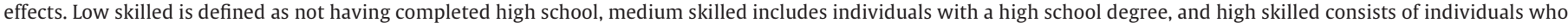
have attended college.

\section{Labor income dynamics}

\subsection{A panel data specification for labor income dynamics}

To understand the role of the tax and transfer system in attenuating shocks to income for individuals and families over the life-cycle requires a model that allows for key aspects in the evolution of labor market income over the working life. As we noted in the introduction, the extensive literature on the panel data modeling of income dynamics has pointed to three key ingredients of potential significance: shocks of varying persistence; age and time dependence in the variance of shocks; and heterogeneous age profiles. The size and detailed nature of the Norwegian population panel allow us to combine all three of these components and let the degree of persistence and the variance of the shocks vary in a quite unrestricted way by age and calendar time.

Let $Y_{i, a}^{c}$ denote the market income of individual $i$ from birth cohort $c$ at age $a$. To obtain log income net of observable characteristics and common aggregate time trends, denoted $y_{i, a}^{c}$, we run cross-sectional firststage regressions of $\log Y_{i, a}^{\mathrm{c}}$ on a set of covariates. ${ }^{11} \mathrm{~A}$ panel data specification that encompasses many of the ideas in the literature is:

$y_{i, a}^{c}=\alpha_{i}^{c}+\beta_{i}^{c} p_{a}+v_{i, a}^{c}+\tau_{i, a}^{c}$,

where $\alpha_{i}^{c}$ is an individual initial condition, while $\beta_{i}^{c}$ allows for an idiosyncratic experience profile in the deterministic trend variable $p_{a}$ (e.g. $p_{a}=a-25$ with a linear experience profile). Taken together, these two terms capture individual-specific unobserved heterogeneity in the levels and growth rates of labor income. We allow for correlation between $\alpha_{i}^{c}$ and $\beta_{i}^{c}$.

Income shocks are decomposed into a permanent (or persistent) component $v_{i, a}^{\mathrm{c}}$,

$v_{i, a}^{c}=\rho^{c} v_{i, a-1}^{c}+u_{i, a}^{c}$,

where $u_{i, a}^{c}$ is a serial uncorrelated mean-zero shock, and a transitory component $\tau_{i, a}^{\mathrm{c}}$, which is assumed to follow a MA(1) process.

$\tau_{i, a}^{c}=\varepsilon_{i, a}^{c}+\theta^{c} \varepsilon_{i, a-1}^{c}$,

where $\varepsilon_{i, a}^{c}$ is a serial uncorrelated mean-zero shock. The permanent and transitory innovations are assumed to be independent of each other and

\footnotetext{
11 In each year, we perform a separate OLS regression of $\log Y_{i, a}^{c}$ on a quadratic polynomial in age and dummies for education, region, family size and marital status. We allow for interactions between family size and marital status as well as interactions between the quadratic polynomial in age and the education dummies. From these regressions, we predict $\log$ market income of individual $i$ from birth cohort $c$ at age $a, \log \hat{Y}_{i, a}^{c}$. The residual log income $y_{i, a}^{c}$ is given by $\log Y_{i, a}^{c}-\log \hat{Y}_{i, a}^{c}$. By conditioning on family size and marital status we may underestimate overall income risk.
}

independent of $\alpha_{i}^{c}$ and $\beta_{i}^{c}$. Some examples of permanent innovations are associated with job mobility, long-term unemployment, health shocks, and promotions. Transitory shocks to individual labor income typically include overtime labor supply, piece-rate compensation, bonuses, etc.; in general, such shocks are mean reverting and their effect does not last long.

In Appendix A: Estimation Details, we describe every step of the estimation procedure for the income process given in (1). There are, however, three important features to notice. First, we allow for the permanent component $v_{i, a}^{c}$ to have a $\rho^{c}$ coefficient less than unity. Since we have long enough panels for individuals in each of the cohorts, the parameters of this process together with those for the transitory process and the heterogeneous profiles can be separately identified.

Second, the overall persistence of shocks to the net log income measure $y_{i, a}^{c}$ depends on the weighted sum of the permanent and transitory processes $v_{i, a}^{c}$ and $\tau_{i, a}^{c}$ respectively, where the weights reflect the variance share of each of these components. To see this, consider our baseline specification of the income process which imposes homogenous experience profiles (i.e. $\beta_{i}^{c}=0$ ). Assuming that $\operatorname{var}\left(y_{i, a}^{c}\right) \simeq \operatorname{var}\left(y_{i, a+1}^{c}\right)$, the first order autocorrelation at age $a$

$\varrho_{a}^{c}=\frac{\operatorname{cov}\left(y_{i, a}^{c}, y_{i, a+1}^{c}\right)}{\sqrt{\operatorname{var}\left(y_{i, a}^{c}\right)} \sqrt{\operatorname{var}\left(y_{i, a+1}^{c}\right)}}$

can be expressed as

$$
\varrho_{a}^{c} \simeq \frac{\operatorname{var}\left(\alpha_{i}^{c}\right)+\rho^{c} \sum_{s=0}\left(\rho^{c}\right)^{2 s} \operatorname{var}\left(u_{i, a-s}^{c}\right)+\theta^{c} \operatorname{var}\left(\varepsilon_{i, a}^{c}\right)}{\operatorname{var}\left(\alpha_{i}^{c}\right)+\sum_{s=0}\left(\rho^{c}\right)^{2 s} \operatorname{var}\left(u_{i, a-s}^{c}\right)+\operatorname{var}\left(\varepsilon_{i, a}^{c}\right)+\left(\theta^{c}\right)^{2} \operatorname{var}\left(\varepsilon_{i, a-1}^{c}\right)} .
$$

This illustrates that by allowing the variances of each component to differ by age, we are in effect, allowing the autocorrelation of income shocks to vary quite unrestrictedly over the life cycle (even though $\rho$ does not depend on age).

Lastly, the use of data that follows actual cohorts over the life cycle allows us to accurately measure their true earnings pattern and estimate the labor income dynamics experienced by individuals. The model given in Eq. (1) is estimated separately by education levels using an equally-weighted minimum distance approach applied to second order moments. At every age, we average the moments across cohorts before estimating the income process. ${ }^{12}$ Without further restrictions, the estimates can be interpreted as an average (or a typical) labor income dynamics experienced by these cohorts over their

\footnotetext{
12 We have also estimated model (1) separately for each cohort, and then computed the weighted average of the parameters across the cohorts. Because this procedure is computationally quite costly, and the estimates are very similar, we only report results for which the moments are averaged across the cohorts before estimation.
} 
a) Low-Skilled

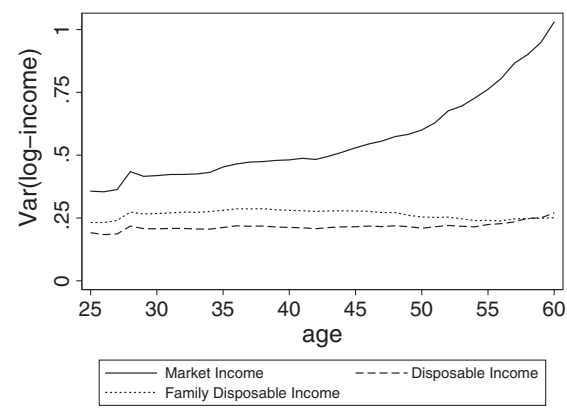

b) Medium-Skilled

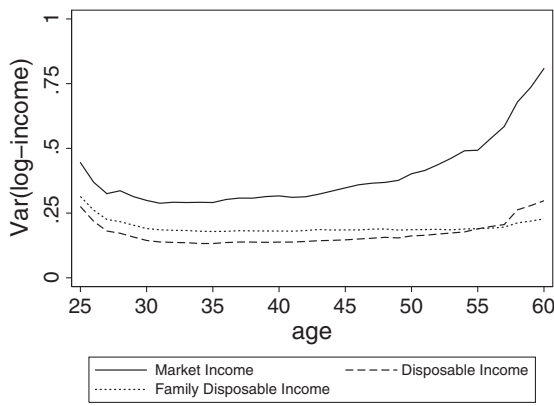

c) High-Skilled

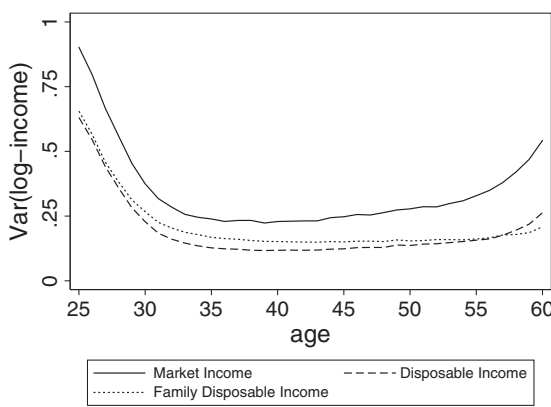

Fig. 2. Age profiles in the variances of log income.

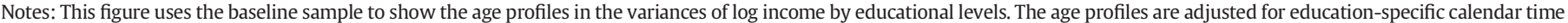

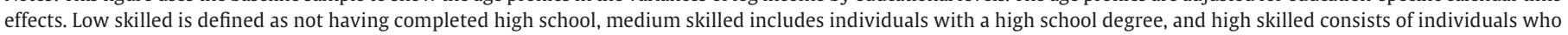
have attended college.

working lifespan. Our focus is on life-cycle effects and to determine the relative contribution of age and calendar time effects to the labor income dynamics we require further restrictions. If one were to assume no cohort effects, we would effectively control for calendar time effects by averaging the moments across cohorts. Heathcote et al. (2005) argue that time effects are required to account for the observed trends in inequality. One might, however, suspect that cohort effects should play some role in the distribution of fixed effects. For example, rising college enrollment rates may have changed the level of permanent wage dispersion of younger relative to older cohorts. We incorporate this source of heterogeneity across cohorts by estimating the income processes separately by education levels.

\subsection{Baseline estimates}

We begin by considering the labor income dynamics of males. The model given in Eq. (1) is estimated separately by education levels. ${ }^{13}$ For now, we impose homogenous experience profiles $\left(\beta_{i}=0\right)$ in the estimation. ${ }^{14}$ Instead of presenting the labor income dynamics of each cohort, we average the moments across the cohorts before estimating the income process. As a result, the estimates should be interpreted as an average (or a typical) labor income dynamics experienced by these cohorts over their working lifespan.

The first column of Table 1 reports the parameter estimates for individual market income of males. For each skill group, we find that the persistence parameter $(\rho)$ is either one or close to one. This suggests that the shocks to $(\log )$ labor income can be described as the sum of a transitory shock and a highly persistent process. Because of the unit root, we do not identify the variance of initial conditions $\left(\operatorname{var}\left(\alpha_{i}\right)\right)$ for the low or medium skilled.

The more persistent the shocks, the more important it is to know whether workers at different ages face the same variance of permanent shocks, or if the magnitude changes systematically over the life-cycle. Fig. 3 examines this by showing the age profile in the variance of permanent shocks $\left(\operatorname{var}\left(u_{i, a}\right)\right)$ according to education levels. The magnitude of permanent shocks varies systematically over the life-cycle. Indeed, we may strongly reject the standard specification with age-independent variance components.

Another key finding is the heterogeneity in the variances of permanent shocks by education levels. For low skilled, the magnitude of permanent shocks is monotically increasing in age. For example, a

\footnotetext{
13 Standard errors are based on nonparametric bootstrap (of each estimation stage) with 70 bootstrap replications.

14 As shown in Section 5, the persistence, magnitude, and age profiles of the permanent and transitory shocks change little when we allow for heterogeneous profiles $\left(\beta_{i}^{c} \neq 0\right)$.
}

permanent shock of one standard deviation implies a 35\% change in individual market income for a low skilled 30 year old; the corresponding number for a low skilled 55 year old is $50 \%$. High skilled, on the other hand, experience large permanent shocks early in life; these shocks decrease in magnitude until age 35 , after which they are relatively small and fairly stable. For example, a permanent shock of one standard deviation implies a $28 \%$ change in individual market income for a high skilled 55 years old; the corresponding number for a high skilled 28 (40) years old is 44 (22) percent.

The variance of transitory shocks, shown in Fig. 4, exhibits a decreasing profile over the life-cycle. While this findings holds for all skill groups, high skilled tend to experience relatively large transitory shocks early in life. At the same time, the MA parameter differs by skill group. A larger proportion of the transient shocks persist for another period for high skilled workers than for low skilled workers.

To see the importance of low incomes in determining the age profiles of labor market shocks, we present results in Appendix Figs. C.6 and C.7 where we exclude observations with low market incomes. ${ }^{15}$ The profiles are much flatter. The presence of low market incomes early and late in life is mirrored in hours of work over the life-cycle. When looking at decennial Norwegian Census data over the period of study, we find that mean hours across the life-cycle is inverse U-shaped. There is an increase until individuals are in their early 30s, then a flattening, and eventually a decrease toward retirement. The opposite is true for the variance of log hours, which is U-shaped. In particular, there is a sharp downward trend in the dispersion of hours worked before age $35 .{ }^{16}$

\subsection{Model fit}

We now examine the performance of the model given in Eq. (1) in fitting the variance of (residual) income growth rates as well as onelag covariances. For each income measure and every skill group, we conclude that the baseline specification with homogenous profiles $\left(\beta_{i}=0\right)$ and a MA(1) achieves a very good fit of these key moments over the lifecycle.

The theoretical moments for the baseline model are given by

$$
\begin{aligned}
\operatorname{var}\left(\Delta y_{i, a}^{c}\right)= & \left(\rho^{c}-1\right)^{2} \operatorname{var}\left(v_{i, a-1}^{c}\right)+\operatorname{var}\left(u_{i, a}^{c}\right)+\operatorname{var}\left(\varepsilon_{i, a}^{c}\right) \\
& +\left(\theta^{c}-1\right)^{2} \operatorname{var}\left(\varepsilon_{i, a-1}^{c}\right)+\theta^{2} \operatorname{var}\left(\varepsilon_{i, a-2}^{c}\right)
\end{aligned}
$$

\footnotetext{
${ }^{15}$ In these figures, we exclude observations with market income lower than the basic amount threshold of the Norwegian Social Insurance Scheme; market income above this threshold gives eligibility for unemployment benefits and matters for old-age pension payments.

${ }^{16}$ Kaplan (2012) documents patterns in the US. He argues that labor market frictions are important in accounting for the patterns of inequality in consumption and hours over the life-cycle.
} 
Table 1

Parameter estimates from the model of income dynamics.

\begin{tabular}{|c|c|c|c|}
\hline & $\begin{array}{l}\text { Individual market } \\
\text { income }\end{array}$ & $\begin{array}{l}\text { Individual disposable } \\
\text { income }\end{array}$ & $\begin{array}{l}\text { Family disposable } \\
\text { income }\end{array}$ \\
\hline \multicolumn{4}{|c|}{ Low-skill } \\
\hline$\rho$ & $\begin{array}{l}1.00 \\
(0.000000)\end{array}$ & $\begin{array}{l}0.87 \\
(0.006287)\end{array}$ & $\begin{array}{l}0.87 \\
(0.003763)\end{array}$ \\
\hline $\operatorname{var}\left(\alpha_{i}\right)$ & - & $\begin{array}{l}0.035360 \\
(0.001234)\end{array}$ & $\begin{array}{l}0.034113 \\
(0.001018)\end{array}$ \\
\hline$\theta$ & $\begin{array}{l}0.238500 \\
(0.004793)\end{array}$ & $\begin{array}{l}0.215220 \\
(0.004638)\end{array}$ & $\begin{array}{l}0.207820 \\
(0.004811)\end{array}$ \\
\hline \multicolumn{4}{|c|}{ Medium-skill } \\
\hline$\rho$ & $\begin{array}{l}1.00 \\
(0.000000)\end{array}$ & $\begin{array}{l}0.89 \\
(0.005500)\end{array}$ & $\begin{array}{l}0.89 \\
(0.004781)\end{array}$ \\
\hline $\operatorname{var}\left(\alpha_{i}\right)$ & $\begin{array}{l}- \\
-\end{array}$ & $\begin{array}{l}0.030796 \\
(0.001162)\end{array}$ & $\begin{array}{l}0.027141 \\
(0.000899)\end{array}$ \\
\hline$\theta$ & $\begin{array}{l}0.258840 \\
(0.0028949)\end{array}$ & $\begin{array}{l}0.238450 \\
(0.003982)\end{array}$ & $\begin{array}{l}0.243650 \\
(0.003916)\end{array}$ \\
\hline \multicolumn{4}{|c|}{ High-skill } \\
\hline$\rho$ & $\begin{array}{l}0.98 \\
(0.013981)\end{array}$ & $\begin{array}{l}0.94 \\
(0.029910)\end{array}$ & $\begin{array}{l}0.85 \\
(0.010652)\end{array}$ \\
\hline $\operatorname{var}\left(\alpha_{i}\right)$ & $\begin{array}{l}0.000152 \\
(0.000043)\end{array}$ & $\begin{array}{l}0.000447 \\
(0.014922)\end{array}$ & $\begin{array}{l}0.030992 \\
(0.000868)\end{array}$ \\
\hline$\theta$ & $\begin{array}{l}0.294650 \\
(0.0049582)\end{array}$ & $\begin{array}{l}0.270220 \\
(0.005652)\end{array}$ & $\begin{array}{l}0.278160 \\
(0.005665)\end{array}$ \\
\hline
\end{tabular}

Notes: This table presents the parameter estimates from the model of income dynamics described in Section 3.1. We use the baseline sample and estimate the model separately by educational levels. Low skilled is defined as not having completed high school, medium skilled includes individuals with a high school degree, and high skilled consists of individuals who have attended college. Standard errors (in parentheses) are based on nonparametric bootstrap (of both estimation stages) with 70 bootstrap replications.

$$
\begin{aligned}
\operatorname{cov}\left(\Delta y_{i, a}^{c}, \Delta y_{i, a+1}^{c}\right)= & \left(\rho^{c}-1\right)^{2} \rho^{c} \operatorname{var}\left(v_{i, a-1}^{c}\right)+\left(\rho^{c}-1\right) \operatorname{var}\left(u_{i, a}^{c}\right) \\
& +\left(\theta^{c}-1\right) \operatorname{var}\left(\varepsilon_{i, a}^{c}\right)-\theta^{c}\left(\theta^{c}-1\right) \operatorname{var}\left(\varepsilon_{i, a-1}^{c}\right) .
\end{aligned}
$$

Appendix Fig. C.10 shows the model fit for the variance of the growth rate, while Appendix Fig. C.11 displays the match for the onelag covariance profile of the growth rate. We find that the model matches the variance of the growth rate observed in the data almost perfectly. When $\rho=1$, we effectively target the variance of the growth rate in the estimation. As a result, the age dependence of the variances shocks allows us to match the age profile very well. When $\rho<1$, the moments used in the estimation differ from those shown in the figure. It is therefore reassuring to find that the model also in this case fits the data very well.

\section{Insurance from taxes, transfers and the family}

The evidence of heterogeneity in the dynamics of labor income by age, skill level, and their interaction raises a number of important questions. To what extent does the tax and transfer system attenuate or insure the shocks to market income at different parts of the life-cycle? Does the addition of income sources from the spouse offset or enhance labor market shocks? In this section, we investigate these questions.

\subsection{Taxes and transfers}

The second column of Table 1 reports the estimation results for individual disposable income of males. Importantly, the tax-transfer system reduces the level and persistence of both the permanent and the transitory shocks. The estimated persistence parameter falls the most for low skilled; when $\rho=0.87$, the effect of an income shock is reduced to $25 \%$ of its initial value in ten years. At the same time, Figs. 3 and 4 show that taxes and transfers lead to a remarkable flattening of the age profiles in the variances of permanent and transitory shocks for the low skilled. At age 55, for example, a permanent shock of one standard deviation implies a 50\% change in annual market income for a low skilled; the corresponding number for annual disposable income is only $31 \%$.

Shifting attention to the high skilled, we can see that taxes and transfers do little to the age profile in the variance of transitory shocks. As shown in Fig. 4, it exhibits a decreasing and convex profile also in individual disposable income; indeed, the magnitudes of the transitory shocks are only slightly lower for disposable income than for market income. The impact of taxes and transfers is somewhat larger for the variance of permanent shocks. Early in life, the permanent shocks to market income of high skilled are attenuated substantially, although they remain large. Toward the end of the life-cycle, the tax-transfer system reduces the magnitude of the permanent shocks somewhat.

Taken together, our results suggest that the redistributive nature of the Norwegian tax-transfer system plays a key role in attenuating the magnitude and persistence of income shocks, especially among the low educated. This finding could have important implications for consumption inequality and the overall ability of families to insure labor income shocks. Economic theory predicts that consumption responds strongly to permanent shocks, and empirical evidence suggests little if any self-insurance in response to permanent shocks among individuals with no college education (see e.g. Blundell et al., 2008).

\subsection{Family income}

We now shift attention to examining whether the addition of income from the spouse offsets or enhances labor market shocks. There a) Low-Skilled

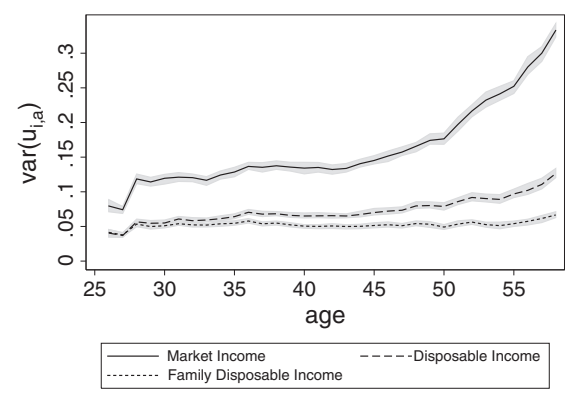

b) Medium-Skilled

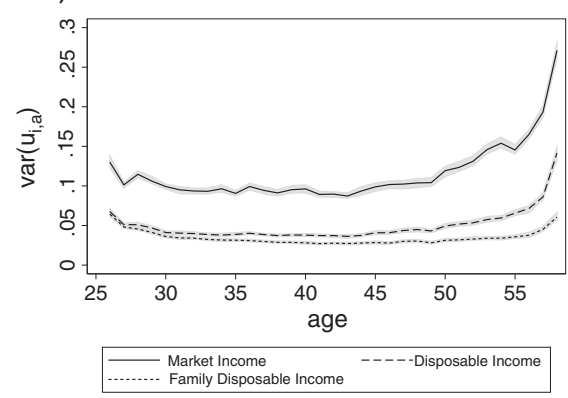

c) High-Skilled

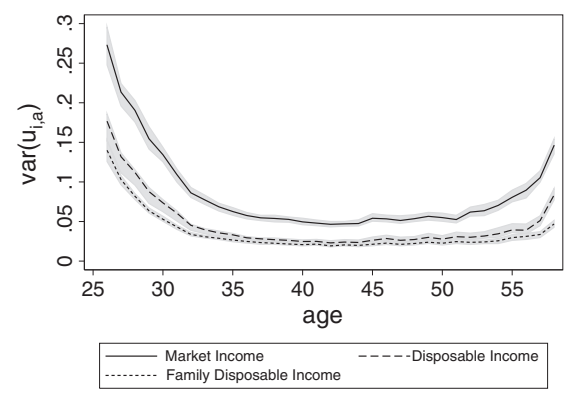

Fig. 3. Age profiles in the variances of permanent shocks to income.

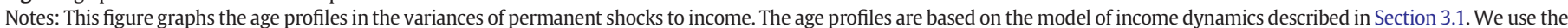

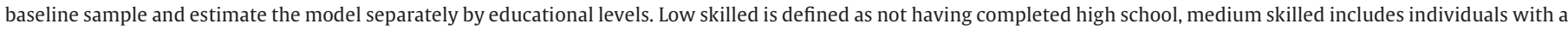

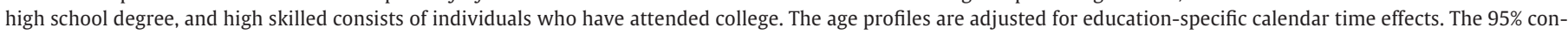
fidence interval is based on nonparametric bootstrap (of both estimation stages) with 70 bootstrap replications. 
a) Low-Skilled

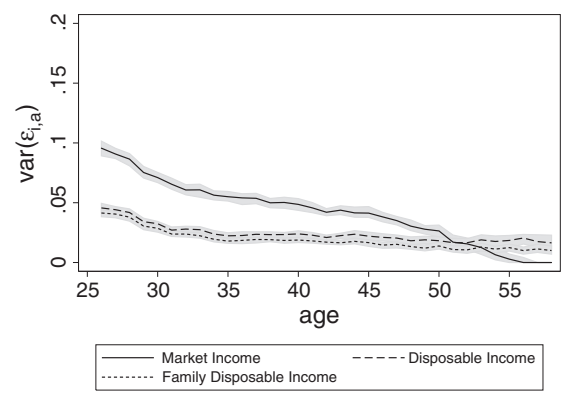

b) Medium-Skilled

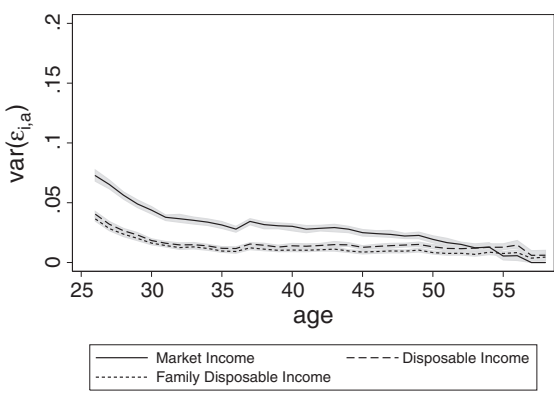

c) High-Skilled

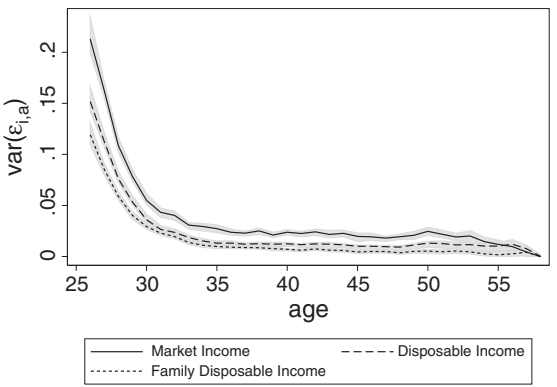

Fig. 4. Age profiles in the variances of transitory shocks to income.

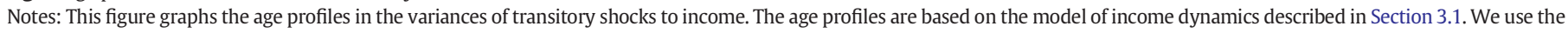

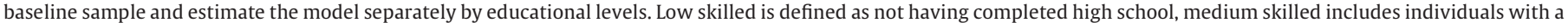

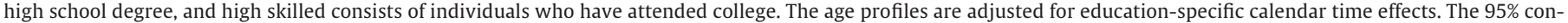
fidence interval is based on nonparametric bootstrap (of both estimation stages) with 70 bootstrap replications.

are competing forces at play when going from individual to family income (see e.g. Blundell et al., 2012). The first is that the variance of market income is relatively large among females, reflecting considerable dispersion in hours worked. The second is that the stochastic components of labor income processes are likely to be correlated across spouses. If spouses were adopting perfect risk sharing mechanisms, they would select jobs where shocks are negatively correlated. Alternatively, assortative mating can imply that spouses work in similar jobs, similar industries, and even in the same firm; as a consequence, their shocks could be positively correlated. The third is that family labor supply is a possible insurance mechanism to market income shocks. For example, the wife's labor supply could increases in response to negative income shocks faced by the husband (see e.g. Lundberg, 1985). By comparing the dynamics of individual and family income over the life cycle, we are able to assess the overall impact of these three factors, but not identify the individual contribution of each factor. ${ }^{17}$

Figs. 3 and 4 display the age profiles in the variances of transitory and permanent shocks to family disposable income. By comparing these profiles to the ones for individual disposable income, we can see that the magnitude of the shocks change little when we add the income of the spouse. This suggests that risk sharing through (negative) assortative mating or labor supply offset the high variance in income of wives and any positively correlated shocks. Table 1 shows that permanent shocks remain highly persistent for low and medium skilled, while falling for the high skilled when we add spouse's income. We can further see that the persistence of transitory shocks change little when including the income of the spouse. ${ }^{18}$

\section{Investigation of income processes}

This section takes advantage of the size and detailed nature of the Norwegian data and brings new evidence on several issues pertinent to the modeling of income processes.

\footnotetext{
17 We have also estimated a model which allows for separate correlation of spouse's permanent and transitory components. To this end, we restrict the baseline sample to couples where both spouses have at least four subsequent observations with positive market income. The estimates from this sample of dual earner couples suggest weak negative correlation across spouses in the shocks to disposable income over most of the life-cycle. However, we need to be cautious in interpreting these estimates because the labor force participation of women is relatively low and unstable; as a result, the dual earner couples are not representative for our baseline sample of working males.

18 Our results are not sensitive to whether we adjust for economies of scale by employing the usual equivalence scales. To see this, note that the log of family income is equal to the $\log$ of the incomes of the husband and the wife, subtracted the log of the equivalence scale (e.g. the square root of family size); the former term will clearly dominate the latter term.
}

\subsection{Nonstationarity in age and time}

Our rich panel data allows us to let the variances components depend on age in an unrestricted way, while controlling flexibly for calendar time effects. This raises questions such as: What is missed by the standard specification in the literature with age-independent variance components? How important is it to account for calendar time effects such as the business cycle or tax reforms?

Appendix Table C.1 investigates the implications of assuming ageindependent shocks. We display the parameter estimates from a model in which the variances of the error components in Eq. (1) are restricted to be constant across the life cycle. For the high skilled, the estimated persistence parameter falls from almost one with age-dependent variance of shocks to .75 with age-independent variance of shocks. By comparison, the age-independent specification does not affect the estimates of the persistence parameter for the low and medium skilled. Figs. 5 and 6 show the misspecification bias from restricting the variances of transitory and permanent shocks to be constant over the life cycle. These figures highlight the importance of allowing for age nonstationarity to capture the labor income dynamics of low and high skilled workers.

What features of the data give rise to the misspecification bias we observe? Recall that high skilled have a U-shaped age profile in the variance of individual market income. We argue that targeting these moments with an age stationary model puts a downward pressure on $\rho$. With a persistent parameter close to one, it becomes difficult to match the U-shaped profile with an age-independent specification because the permanent shocks would then accumulate over the life cycle, generating an increasing and convex profile in the variance of individual market income.

In Fig. 7, we illustrate the importance of allowing for time nonstationarity to get a clear picture of the typical income dynamics over the life cycle. We estimate the model given in (1) separately by cohort, and graph the age profiles in the variance of transitory shocks to disposable income for different cohorts; for brevity, we do not split the sample by education. The 5 year interval between the cohorts allows us to clearly see the impact of a tax reform: For each cohort, we observe a spike in the variance of transitory shocks at that the time of the change in tax policy. ${ }^{19}$ By comparison, our baseline results control for such calendar time effects by averaging the moments across the cohorts before estimating the income process. After taking out calendar time effects, the variance of transitory shocks exhibits a smooth and decreasing profile over the life-cycle.

\footnotetext{
19 The tax reform affected both the timing of income reporting and the incentives to shift and reclassify income.
} 
a) Low-Skilled

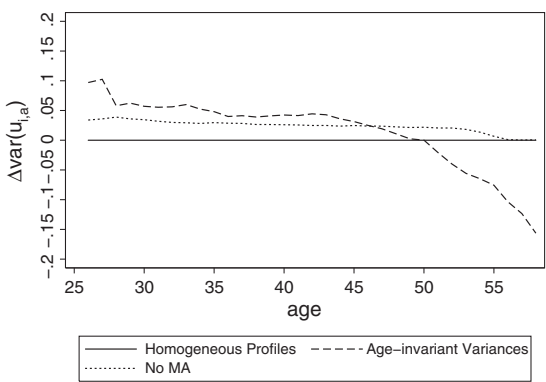

b) Medium-Skilled

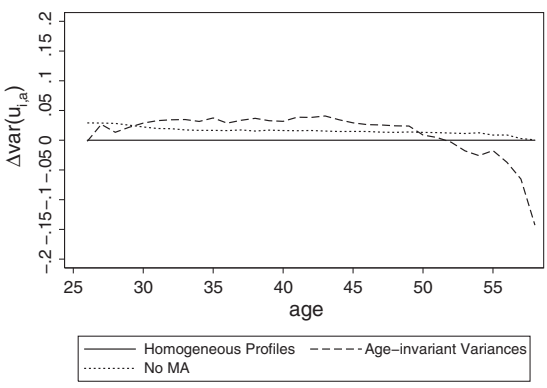

c) High-Skilled

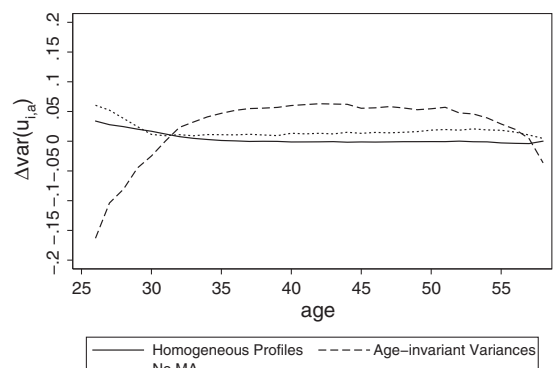

Nomog

Fig. 5. Misspecification bias in the variance of permanent shocks to individual market income.

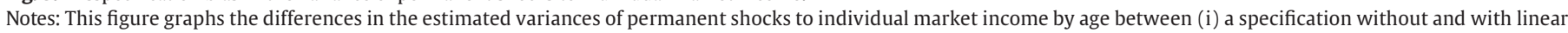

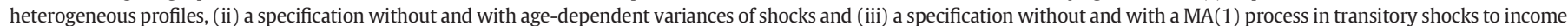

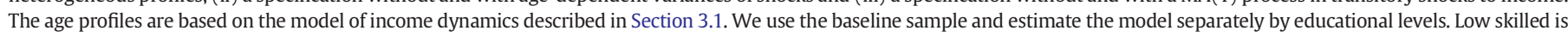

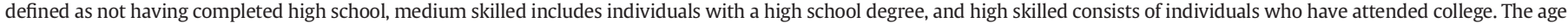
profiles are adjusted for education-specific calendar time effects.

\subsection{Heterogeneous profiles}

Our findings suggest important heterogeneity in labor income dynamics by age, skill level, and their interaction. This raises questions such as: What happens if we do not allow for the possibility that individuals with different education levels face different income processes at the variance level? How important is it to allow for unobserved heterogeneity in the income growth rates within skill groups?

Appendix Table C.2 displays parameter estimates from the baseline model of income dynamics when we do not split the sample by education. The persistence parameter in the pooled sample is one, suggesting that the shocks to $(\log )$ labor income can still be described as the sum of a transitory shock and a highly persistent process. Figs. C. 8 and C.9 show the age profiles in the variances of shocks when we restrict the income processes at the variance level to be the same across skill groups. Because the results from the pooled sample mix the income processes of low and high skilled, we obtain an inverse U-shaped age profile in the variances of permanent shocks. However, this pattern is at odds with the age profiles of both high and low skilled: While the former group experience large permanent shocks early in life, the latter group faces the largest shocks at older ages. These findings point to the importance of allowing for heterogeneity by education levels to capture the labor income dynamics of young and old workers.
So far, we have imposed homogenous experience profiles (i.e. $\beta_{i}=0$ ) within each skill group. We now relax this assumption and allow for a linear experience profile in the model given by Eq. (1). Appendix Table C.3 displays the parameter estimates for individual market income, while Figs. 5 and 6 show the misspecification bias from imposing homogenous experience profiles. The results suggest education levels do a good job in capturing heterogeneity in the dynamics of labor income over the life-cycle. Only for the high skilled, there is evidence of significant unobserved heterogeneity in the income growth rates; accounting for this heterogeneity lowers the persistent parameter from .98 to .90 , but barely moves the age profiles in the variances of permanent and transitory shocks.

Appendix Fig. C.12 illustrates the heterogeneity in market income profiles for the high skilled. There is a non-negligible fanning out of the income profiles. At the same time, there is a negative correlation between the initial conditions and the individual-specific income growth rate. This means that high skilled workers with relatively low market income at age 25 (the initial age) tend to have stronger income growth over the life cycle, offsetting some of the fanning out displayed in Fig. C.12.

\subsection{Serially correlated transitory shocks}

Because we have long panel of individuals, we can separately identify a transitory process with serially correlated shocks and a permanent a) Low-Skilled

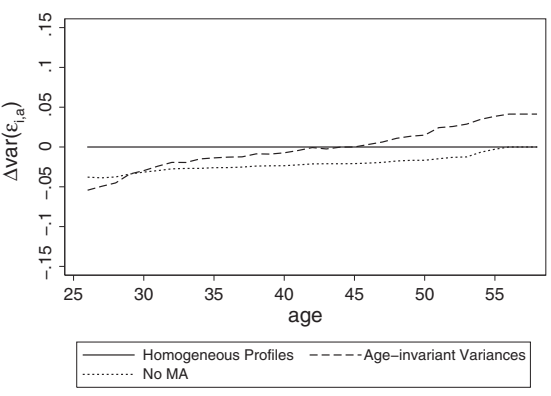

b) Medium-Skilled

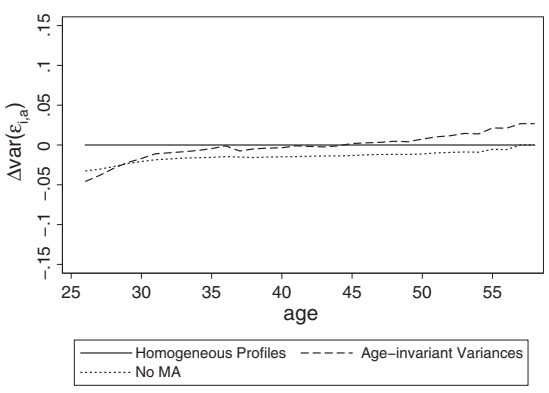

c) High-Skilled

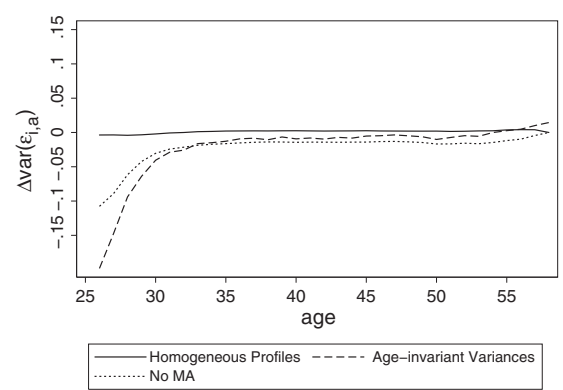

Fig. 6. Misspecification bias in the variance of transitory shocks to individual market income.

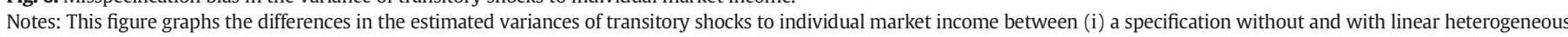

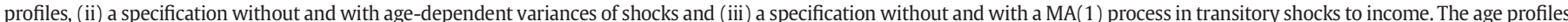

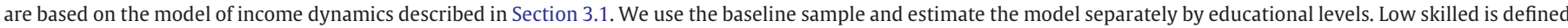

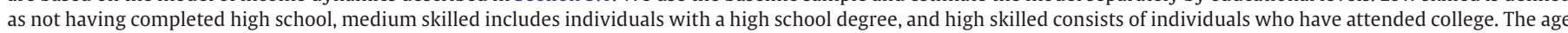
profiles are adjusted for education-specific calendar time effects. 


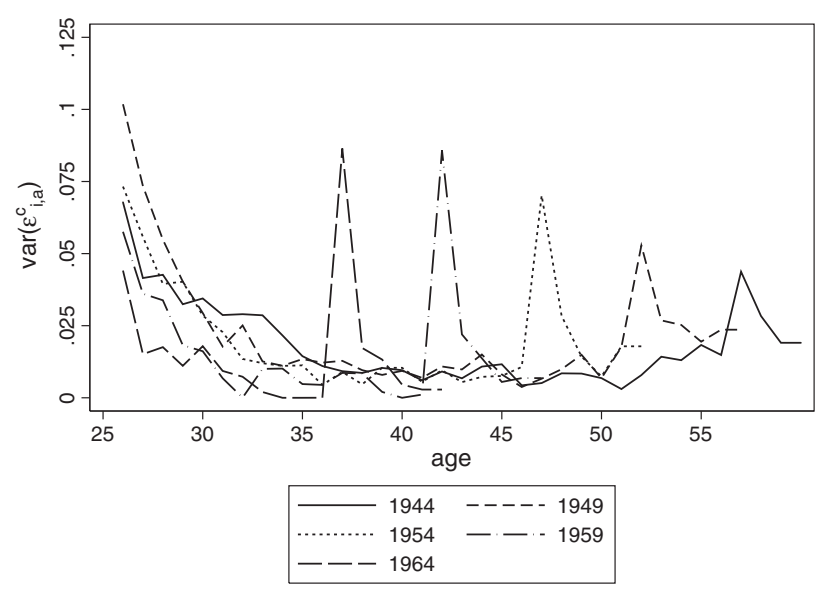

Fig. 7. Age profiles in the variances of transitory shocks to individual disposable income by birth cohort.

Notes: This figure graphs the age profiles in the variances of transitory shocks to individual disposable income separately by cohort. We consider cohorts born in 1944, 1949, 1954, 1959, and 1964. For each cohort, we estimate the model of income dynamics described in Section 3.1.

process which allows for a persistence parameter less than unity. In many cases, however, this is difficult because the panel of individuals is too short (or plagued by problems such as attrition and small sample sizes).

In Figs. 5 and 6, we examine the implications of restricting the transitory component in the model given by Eq. (1) to be uncorrelated over time. In the simple case of serially uncorrelated transitory shocks, all the persistence in the income data is attributed to the permanent income component. By comparison, the transitory component is assigned a larger share of the total variance in our baseline model, because the process captures short-duration persistence in the data. However, our estimates suggest the misspecification bias from assuming serially uncorrelated transitory shocks is relatively small compared to the biases from ignoring heterogeneity in labor income dynamics by age and skill level.

\section{Conclusion}

What do labor income dynamics look like over the life-cycle? What is the relative importance of persistent shocks, transitory shocks and idiosyncratic trends? To what extent do taxes, transfers and the family attenuate these various factors in the evolution of life-cycle inequality? In this paper, we used rich Norwegian panel data to answer these important questions. We estimated a process for income dynamics that allows for key aspects in the evolution of labor income over the life-cycle, including non-stationarity in age and time, shocks of varying persistence, and heterogeneous profiles.

Our estimates of the labor income dynamics of males showed that the magnitude of permanent and transitory shocks varies systematically over the life-cycle, and that there is essential heterogeneity in the variances of these shocks across skill groups. We found that the redistributive nature of the Norwegian tax-transfer system plays a key role in attenuating the magnitude and persistence of income shocks, especially among the low skilled. Spouse's labor market income, on the other hand, matters less for the dynamics of inequality over the life-cycle.

The size and detailed nature of the data we are using also allowed us to bring new evidence on several additional issues pertinent to modeling of income processes. One key finding was that restricting the age and time dependence of the variance of income shocks can lead to quite misleading conclusions about the income process. Another key finding was that allowing for heterogeneity by education levels is necessary to capture the labor income dynamics of young and old workers. By way of comparison, the dynamics of income over the lifecycle change little when restricting the transitory shocks to be uncorrelated over time or allowing for heterogeneous experience profiles within each skill group. Indeed, only for the high skilled, there is evidence of significant unobserved heterogeneity in the income growth rates. Accounting for this heterogeneity lowers the persistence of permanent shocks somewhat, but barely moves the age profiles in the variances of permanent and transitory shocks.

\section{Acknowledgments}

We thank Manuel Arellano, Stéphane Bonhomme and Jean-Marc Robin for many helpful discussions. We are grateful for useful comments from two anonymous referees and the participants at the AEA 2014 meetings in Philadelphia and the Conference on the Nordic Model at the University of Oslo. We are grateful for support from the ESRC Centre for the Microeconomic Analysis of Public Policy at IFS and the Norwegian Research Council.

\section{Appendix A. Estimation details}

\section{A.1. Quasi-differences}

Let $\Delta^{\rho} y_{i, a}^{c} \equiv y_{i, a}^{c}-\rho^{c} y_{i, a}^{c}-1$. Specification (1) implies that

$$
\begin{aligned}
\Delta^{\rho} y_{i, a}^{c}= & \alpha_{i}^{c}\left(1-\rho^{c}\right)+\beta_{i}^{c} \Delta^{\rho} p_{a}+u_{i, a}^{c}+\Delta^{\rho} \varepsilon_{i, a}^{c} \\
& +\theta^{c} \Delta^{\rho} \varepsilon_{i, a-1}^{c}, a=a_{\min }^{c}+1, \ldots, a_{\max }^{c}
\end{aligned}
$$

where the youngest and oldest age at which we observe cohort $c$ is denoted by $a_{\min }^{c}$ and $a_{\max }^{c}$ respectively. With $A^{c} \equiv a_{\max }^{c}-a_{\min }^{c}$ we can rewrite Eq. (2) in vectorized form as

$\Delta^{\rho} y_{i}^{c}=\alpha_{i}^{c}\left(1-\rho^{c}\right) \iota+\beta_{i}^{c} \Delta^{\rho} p+u_{i}^{c}+\Delta^{\rho} \varepsilon_{i}^{c}+\theta^{c} \Delta^{\rho} L \varepsilon_{i}^{c}$,

where $\iota$ is a $A^{\mathrm{c}} \times 1$ vector of ones and $L$ represents the lag-operator. The $A^{\mathrm{c}} \times A^{\mathrm{c}}$ auto-covariance matrix is then given by

$$
\begin{aligned}
\operatorname{var}\left(\Delta^{\rho} y_{i}^{c}\right)= & {\left[\left(1-\rho^{c}\right) \iota, \Delta p\right] \operatorname{var}\left(\gamma_{i}^{c}\right)\left[\left(1-\rho^{c}\right) \iota, \Delta p\right]^{\prime}+\operatorname{var}\left(u_{i}^{c}\right) } \\
& +\operatorname{var}\left(\Delta^{\rho} \varepsilon_{i}^{c}+\theta^{c} \Delta^{\rho} L \varepsilon_{i}^{c}\right),
\end{aligned}
$$

where we have used the notation $\gamma_{i}^{c} \equiv\left[\alpha_{i}^{c}, \beta_{i}^{c}\right]^{\prime 20}$

\section{A.2. Estimation}

For a given value of $\rho$ and for each cohort $c$, we calculate the empirical counterpart to expression (4). We then average the $\left(a, a^{\prime}\right)$-cell of these matrices across all cohorts that we jointly observe at age $a$ and age $a^{\prime}$. Let the resulting empirical auto-covariance matrix be denoted by $\operatorname{var}\left(\Delta^{\rho} y_{i}\right)$ and define the stacked vector of its unique elements by

$$
\left.\hat{M}=\operatorname{vech}\left(\widehat{\operatorname{var}\left(\Delta^{\rho}\right.} y_{i}\right)\right)
$$

\footnotetext{
${ }^{20}$ Note that the $(a, a+s)$ element of $\operatorname{var}\left(\Delta^{\rho} y_{i}^{c}\right)$ is given by

$\operatorname{cov}\left(\Delta^{\rho} y_{i, a}^{c}, \Delta^{\rho} y_{i, a+s}^{c}\right)=\left[\left(1-\rho^{c}\right), \Delta p_{a}\right] \operatorname{var}\left(\gamma_{i}^{c}\right)\left[\left(1-\rho^{c}\right), \Delta p_{a+s}\right]^{\prime}$$$
+\left\{\begin{array}{cc}
\operatorname{var}\left(u_{i, a}^{c}\right)+\operatorname{var}\left(\varepsilon_{i, a}^{c}\right)+\left(\theta^{c}-\rho^{c}\right)^{2} \operatorname{var}\left(\varepsilon_{i, a-1}^{c}\right)+\left(\theta^{c} \rho^{c}\right)^{2} \operatorname{var}\left(\varepsilon_{i, a-2}^{c}\right) & \text { if } s=0 \\
\left(\theta^{c}-\rho^{c}\right)\left(\operatorname{var}\left(\varepsilon_{i, a,}^{c}\right)-\theta^{c} \rho^{v} \operatorname{var}\left(\varepsilon_{i, a-1}^{c}\right)\right) & \text { if } s=1 \\
-\left(\theta^{c} \rho^{c} \operatorname{var}\left(\varepsilon_{i, a}^{c}\right)\right) & \text { if } s=2 .
\end{array} .\right.
$$ 
Let the parameters to be estimated be denoted by $\Theta$. The equallyweighted minimum distance estimator $\widetilde{\Theta}$ is then given by

$\widetilde{\Theta}=\arg \min _{\Theta}[M(\Theta ; \rho)-\hat{M}][M(\Theta ; \rho)-\hat{M}]$,

where $M(\Theta ; \rho)$ are the corresponding stacked vector of theoretical moments. Since we have averaged the empirical moments across cohorts, $\Theta$ contains the average (or typical) profiles of variances of permanent and transitory shocks, $\operatorname{var}\left(u_{i}\right)$ and $\operatorname{var}\left(\varepsilon_{i}\right)$, the moving average parameter $\theta$, an estimate for the typical variance of initial conditions $\operatorname{var}\left(\alpha_{i}\right)$, the variance of the growth rate $\operatorname{var}\left(\beta_{i}\right)$ and the correlation between $\alpha_{i}$ and $\beta_{i}$ denoted by $\rho_{\alpha \beta}$. Once we have solved for $\widetilde{\Theta}$ for each value of $\rho$ belonging to a grid, we select the estimator $\widetilde{\Theta}$ together with $\rho$ that minimizes the distance between the empirical and theoretical moments. ${ }^{21}$ Finally, we perform this estimation separately by education and income measures.

\section{Appendix B. The tax system}

The Norwegian tax system is progressive through deductions and surtaxes. Fig. B.1 shows the marginal tax rates for single earner couples and for single persons (or dual earner couples) at the end of 2006. There is a $7.8 \%$ social security contribution on market income. The market income is taxed at a flat rate of $28 \%$; on top of that, there are two surtax brackets adding an additional 9 and $12 \%$ to the marginal tax rates. Single earner couples and single persons (or dual earner couples) are taxed differently: The latter type of households only gets $50 \%$ of the standard deduction. Over time, the Norwegian tax system has become less progressive through a series of policy changes. Fig. B.2 summarizes these changes by displaying the average tax rates on market income over time.

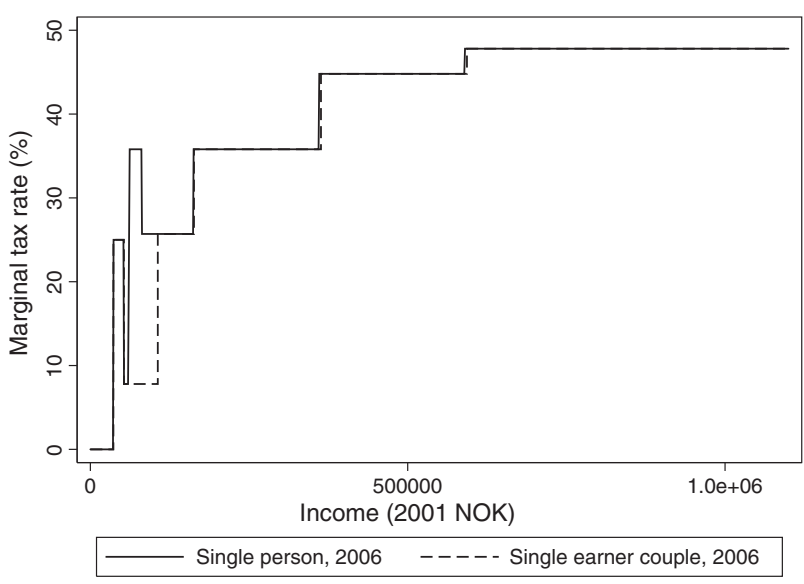

Fig. B.1. Marginal tax rates on market income in 2006

\footnotetext{
${ }^{21}$ In practice, we find $\widetilde{\Theta}$ for a given value of $\rho$ by solving a constrained nonlinear optimization problem. The inequality constraints imposed ensure positive values for variances at any age. We further normalize the variance of transitory shocks to be constant from age 24 to age 26 and the variance of permanent and transitory shocks to be constant between ages 59 and 60 . Note that in our baseline specification we also impose $\beta_{i}=0$.
}
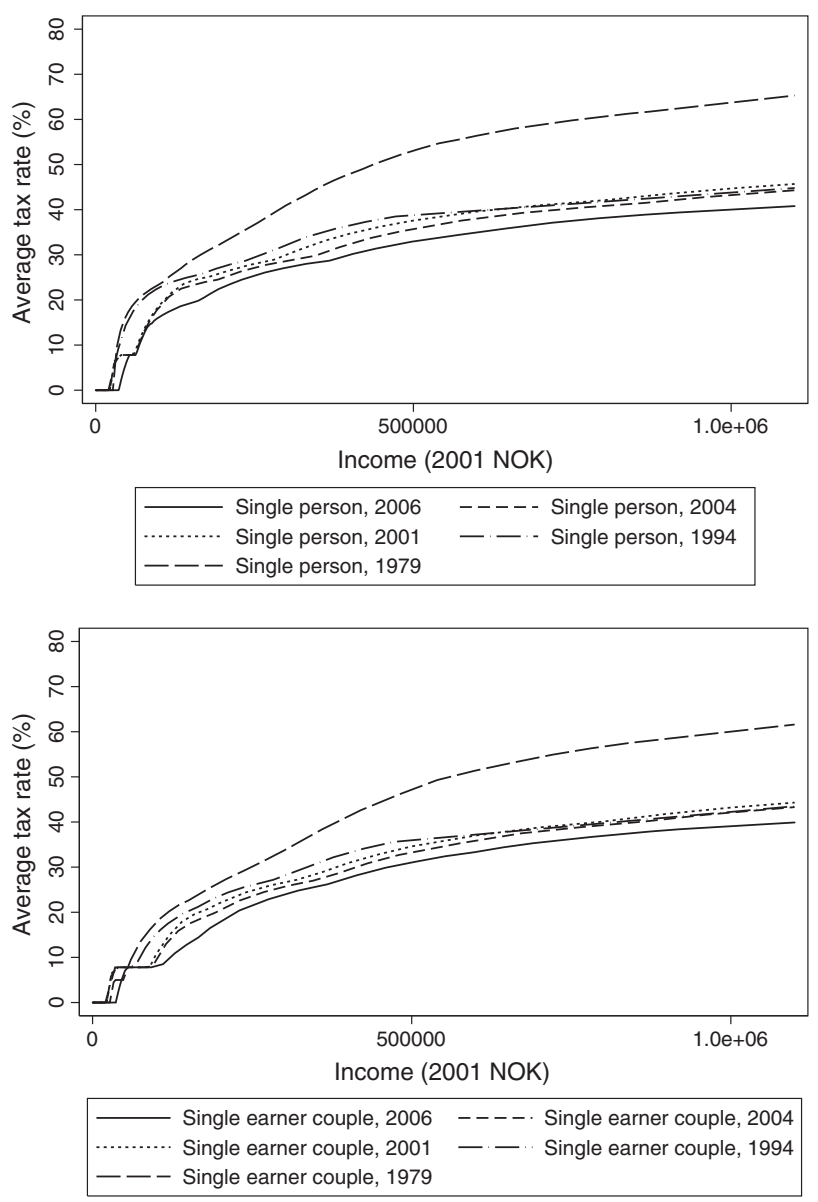

Fig. B.2. Average tax rates on market income in different years.

\section{Appendix C. Additional tables and figures}

Table C.1

Parameter estimates from the model of income dynamics with age-independent variance of shocks.

\begin{tabular}{llll}
\hline & Low-skilled & Medium-skilled & High-skilled \\
\hline$\rho$ & 1.00 & 1.00 & 0.75 \\
$\operatorname{var}\left(\alpha_{i}\right)$ & $(0.000000)$ & $(0.000000)$ & $(0.000000)$ \\
& - & - & 0.077078 \\
$\operatorname{var}\left(u_{i}\right)$ & - & - & $(0.000753)$ \\
& 0.176590 & 0.128030 & 0.109590 \\
$\operatorname{var}\left(\varepsilon_{i}\right)$ & $(0.000786)$ & $(0.000530)$ & $(0.000736)$ \\
$\theta$ & 0.041355 & 0.026831 & 0.014493 \\
$\theta$ & $(0.000470)$ & $(0.000332)$ & $(0.000500)$ \\
& 0.289020 & 0.295160 & 0.306340 \\
& $(0.003764)$ & $(0.003180)$ & $(0.009686)$
\end{tabular}

Notes: This table presents the parameter estimates from the model of income dynamics. We estimate the model described in Section 3.1, except for imposing age-independent variances of transitory and permanent shocks. We use the baseline sample and estimate the model separately by educational levels. Low skilled is defined as not having completed high school, medium skilled includes individuals with a high school degree, and high skilled consists of individuals who have attended college. Standard errors (in parentheses) are based on nonparametric bootstrap (of both estimation stages) with 70 bootstrap replications. 
Table C.2

Parameter estimates from the model of income dynamics in the pooled sample.

\begin{tabular}{|c|c|c|c|}
\hline & Individual market income & Individual disposable income & Family disposable income \\
\hline \multicolumn{4}{|l|}{ Pooled } \\
\hline$\rho$ & $\begin{array}{l}1.00 \\
(0.000000)\end{array}$ & $\begin{array}{l}0.85 \\
(0.004133)\end{array}$ & $\begin{array}{l}0.86 \\
(0.004962)\end{array}$ \\
\hline $\operatorname{var}\left(\alpha_{i}\right)$ & - & $\begin{array}{l}0.035644 \\
(0.000483)\end{array}$ & $\begin{array}{l}0.031509 \\
(0.000473)\end{array}$ \\
\hline$\theta$ & $\begin{array}{l}0.271470 \\
(0.001784)\end{array}$ & $\begin{array}{l}0.250870 \\
(0.002389)\end{array}$ & $\begin{array}{l}0.251930 \\
(0.002673)\end{array}$ \\
\hline
\end{tabular}

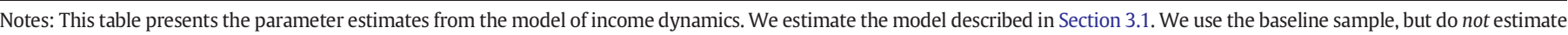
the model separately by educational levels. Standard errors (in parentheses) are based on nonparametric bootstrap (of both estimation stages) with 70 bootstrap replications.

\section{a) Low-Skilled}

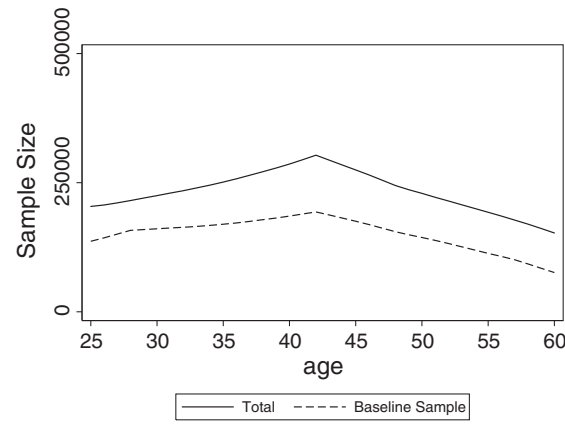

b) Medium-Skilled

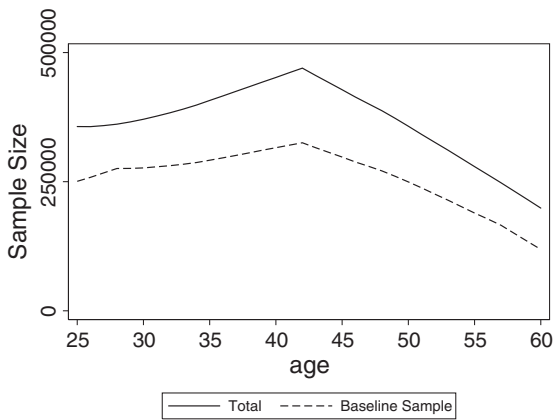

c) High-Skilled

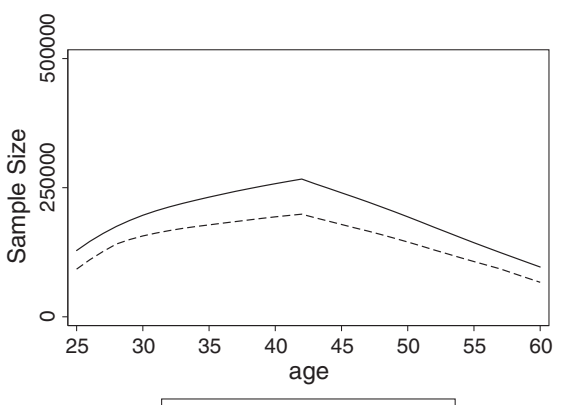

Fig. C.1. Population and sample size by age.

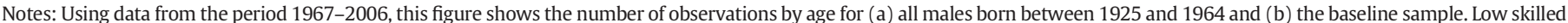
is defined as not having completed high school, medium skilled includes individuals with a high school degree, and high skilled consists of individuals who have attended college.

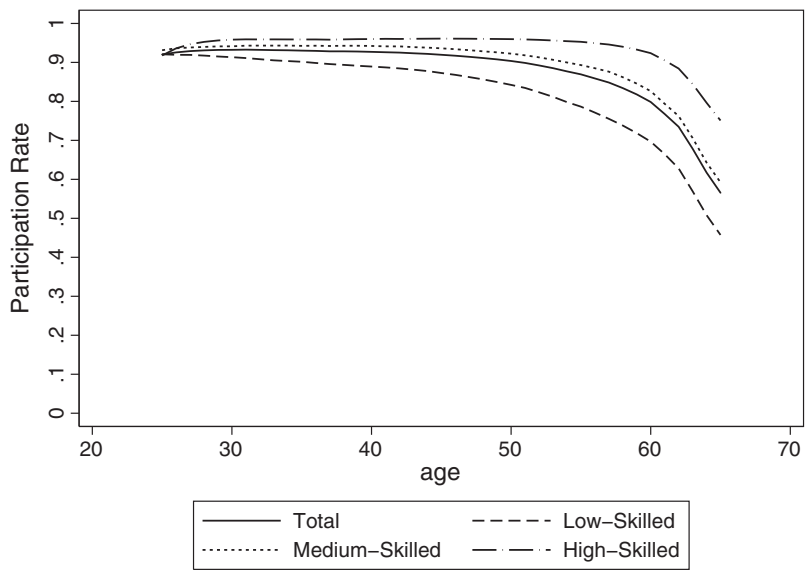

Fig. C.2. Labor force participation rate of males by age.

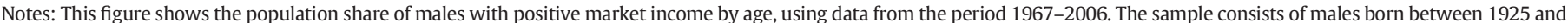

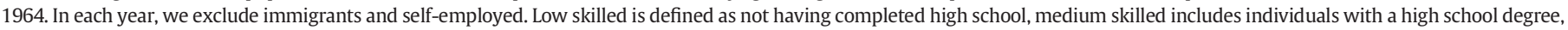
and high skilled consists of individuals who have attended college.

a) Low-Skilled

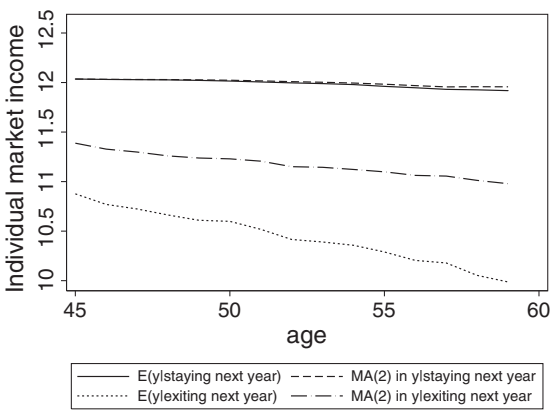

b) Medium-Skilled

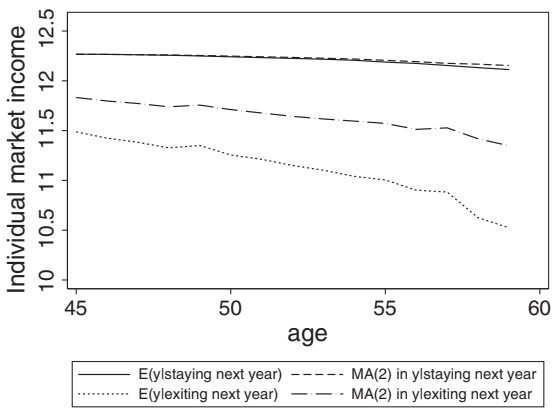

c) High-Skilled

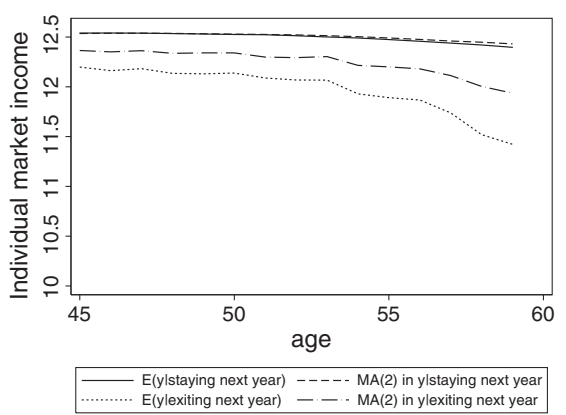

Fig. C.3. Levels and growth in log market income for early and late exits from the labor force.

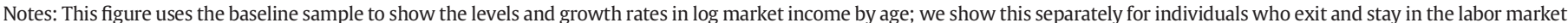

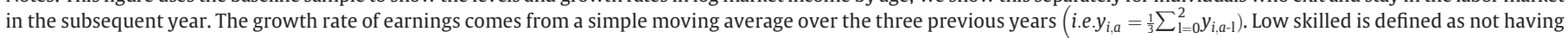
completed high school, medium skilled includes individuals with a high school degree, and high skilled consists of individuals who have attended college. 

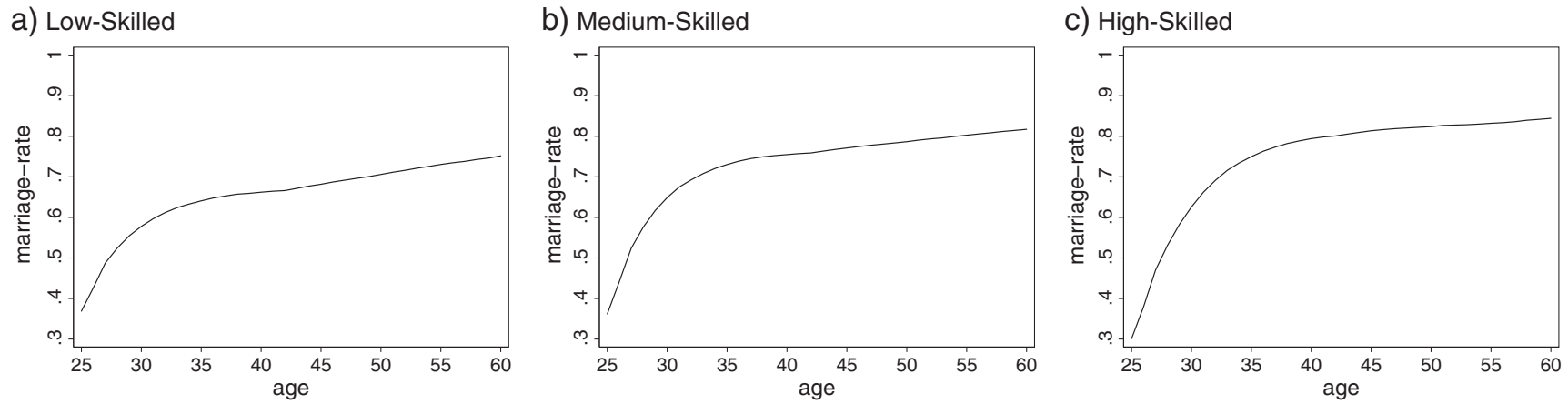

Fig. C.4. Proportion of baseline sample that is married by age and educational levels.

\section{a) Low-Skilled}

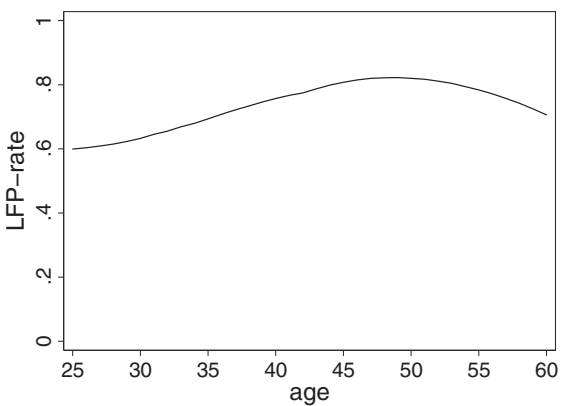

b) Medium-Skilled

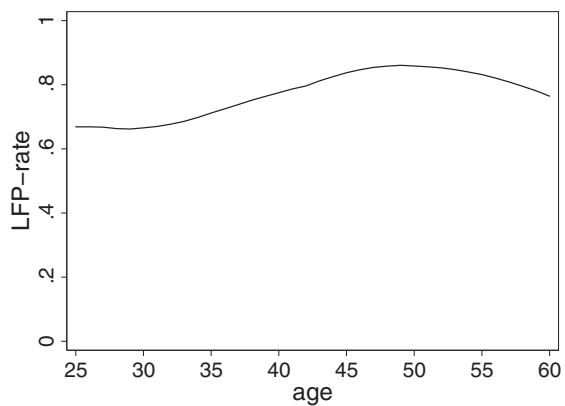

c) High-Skilled

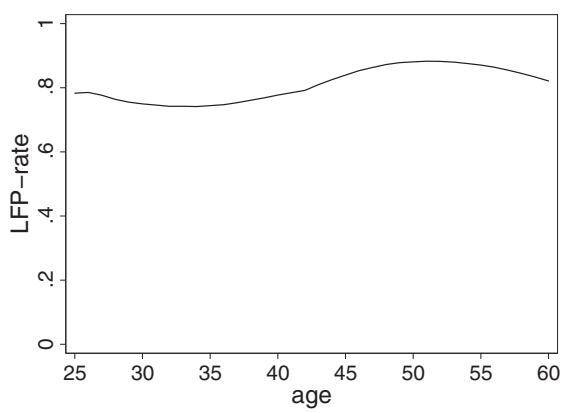

Fig. C.5. Labor force participation rate of spouses by age and educational levels.

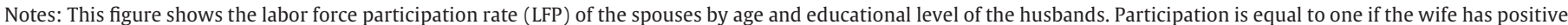
labor income in a given year. This figure uses the baseline sample and, in each year, excludes individuals who are not married.

a) Low-Skilled

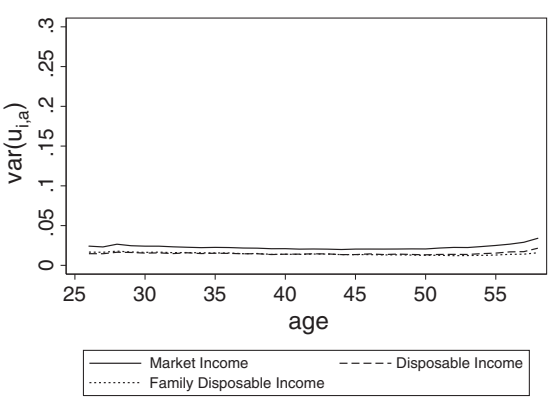

b) Medium-Skilled

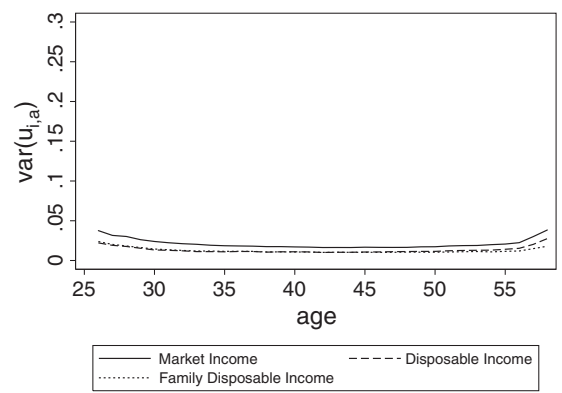

c) High-Skilled

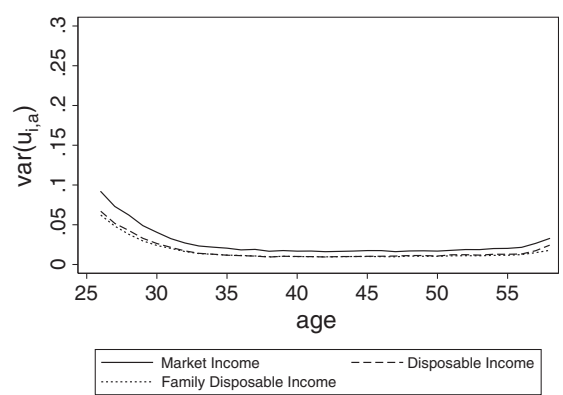

Fig. C.6. Age profiles in the variance of permanent shocks to individual income when excluding individuals with low market income.

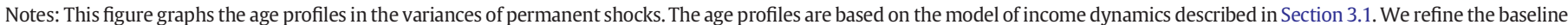

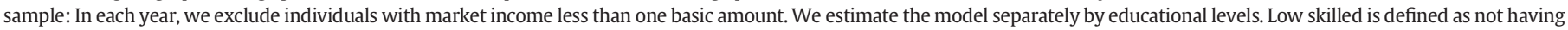

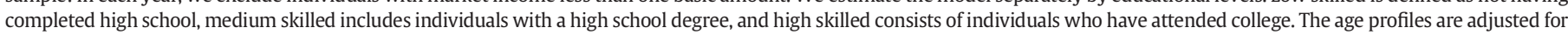
education-specific calendar time effects. 
a) Low-Skilled

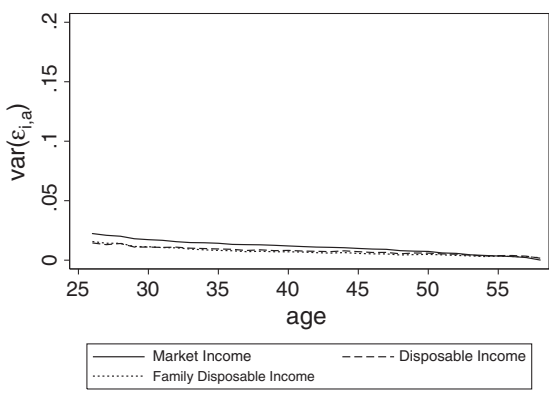

b) Medium-Skilled

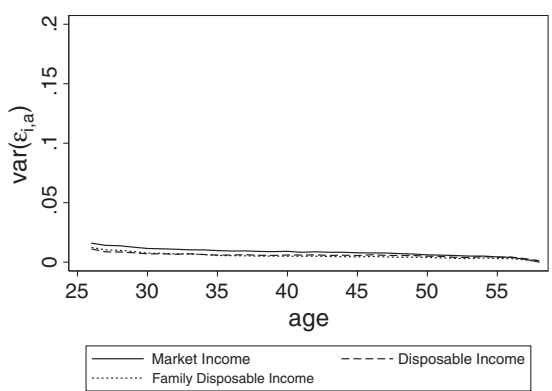

c) High-Skilled

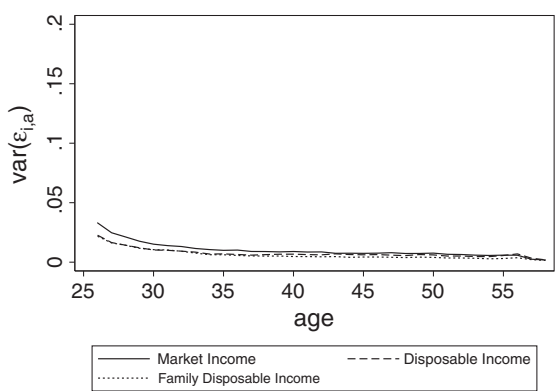

Fig. C.7. Age profiles in the variance of transitory shocks to individual income when excluding individuals with low market income.

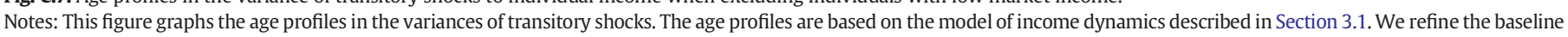

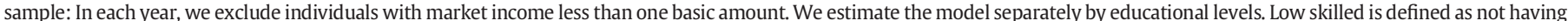

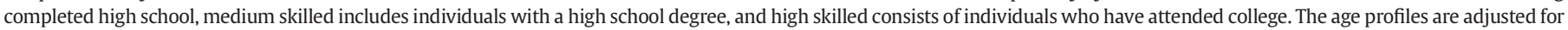
education-specific calendar time effects.

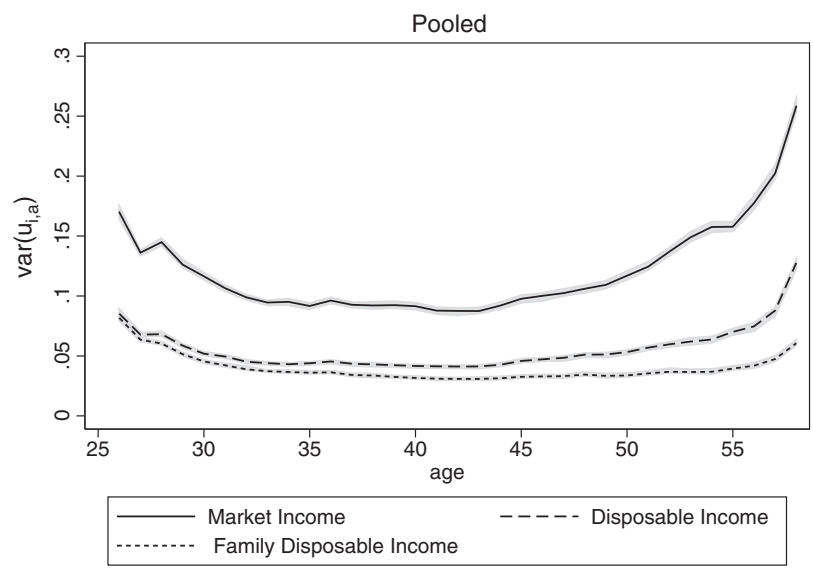

Fig. C.8. Age profiles in the variances of permanent shocks in the pooled sample.

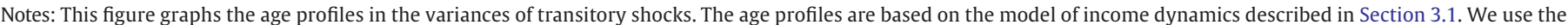

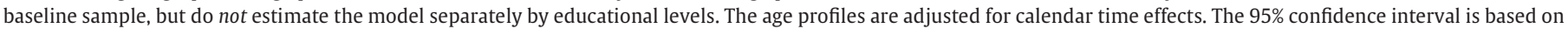
nonparametric bootstrap (of both estimation stages) with 70 bootstrap replications.

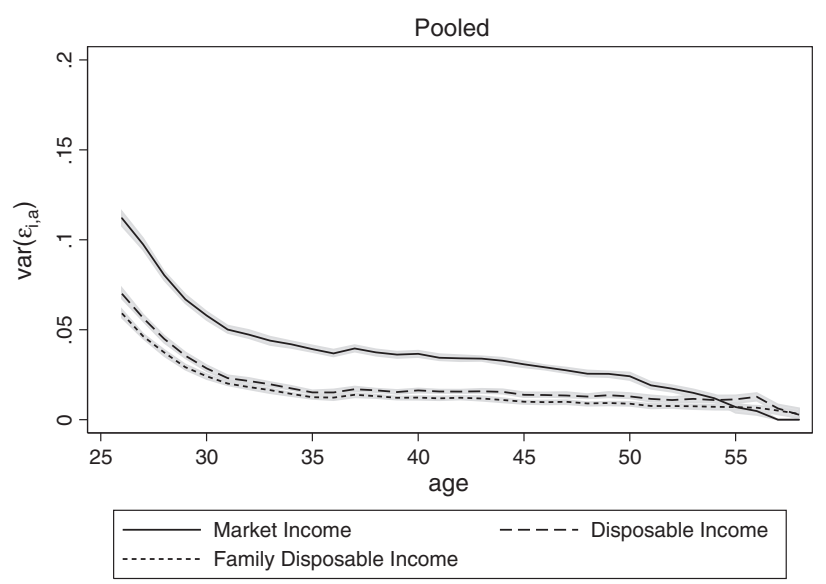

Fig. C.9. Age profiles in the variances of transitory shocks in the pooled sample.

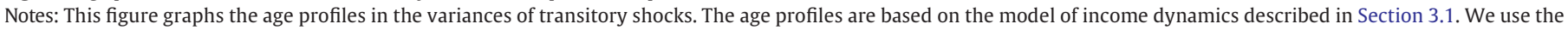

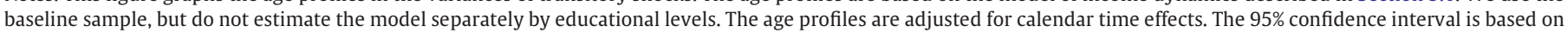
nonparametric bootstrap (of both estimation stages) with 70 bootstrap replications. 
Table C.3

Parameter estimates from the model of income dynamics with heterogeneous profiles in market income.

\begin{tabular}{|c|c|c|c|}
\hline & Low-skilled & Medium-skilled & High-skilled \\
\hline$\rho$ & $\begin{array}{l}1.00 \\
(0.000000)\end{array}$ & $\begin{array}{l}1.00 \\
(0.000000)\end{array}$ & $\begin{array}{l}0.90 \\
(0.045857)\end{array}$ \\
\hline $\operatorname{var}\left(\alpha_{i}\right)$ & - & - & $\begin{array}{l}0.026887 \\
(0.032780)\end{array}$ \\
\hline $\operatorname{var}\left(\beta_{i}\right)$ & $\begin{array}{l}0.000000 \\
(0.000000)\end{array}$ & $\begin{array}{l}0.000000 \\
(0.000000)\end{array}$ & $\begin{array}{l}0.0002773 \\
(0.000098)\end{array}$ \\
\hline$\rho_{\alpha \beta}$ & - & - & $\begin{array}{l}-0.998930 \\
(0.067835)\end{array}$ \\
\hline$\theta$ & $\begin{array}{l}0.238500 \\
(0.003588)\end{array}$ & $\begin{array}{l}0.258830 \\
(0.002895)\end{array}$ & $\begin{array}{l}0.293430 \\
(0.004917)\end{array}$ \\
\hline
\end{tabular}

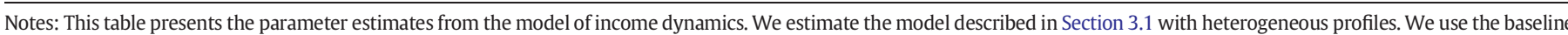

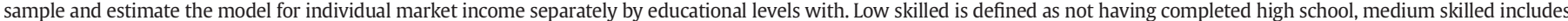

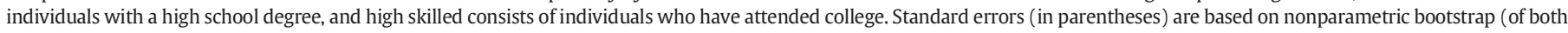
estimation stages) with 70 bootstrap replications.

a) Low-Skilled

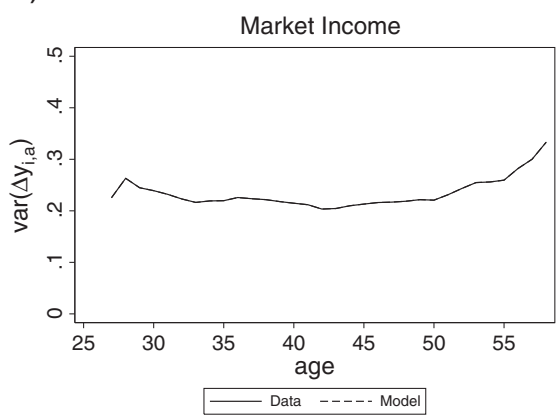

b) Medium-Skilled

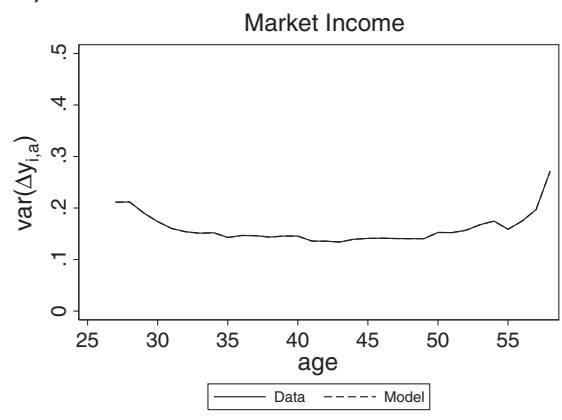

c) High-Skilled

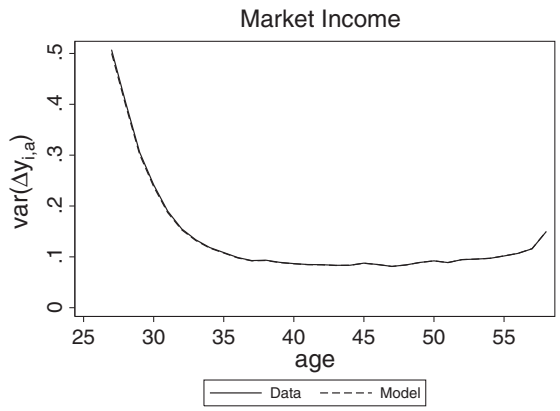

Disposable Income
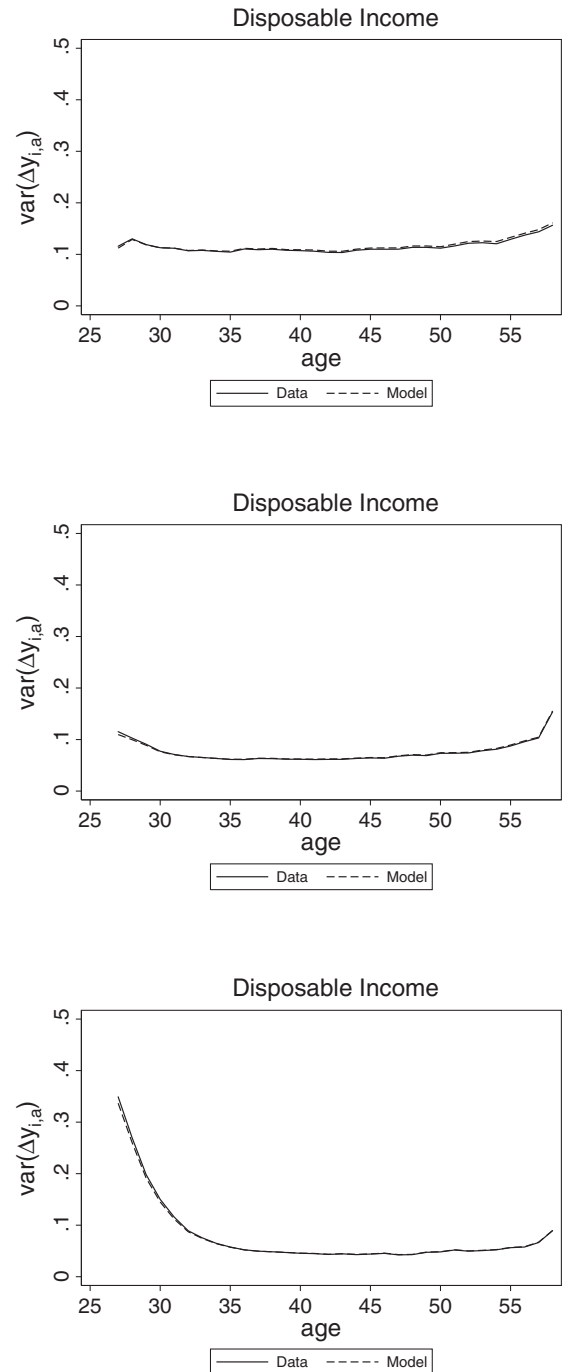

Family Disposable Income

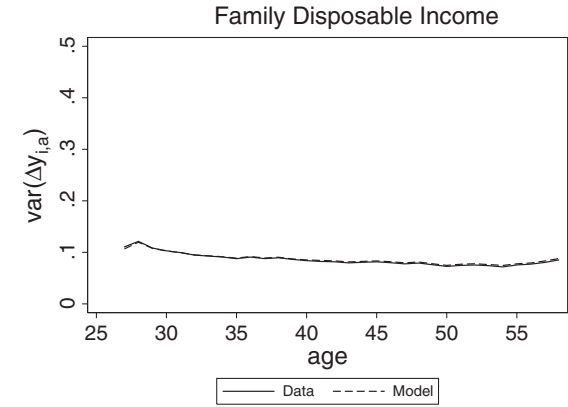

Family Disposable Income

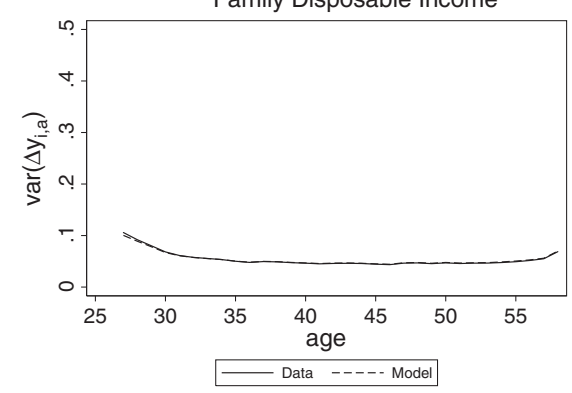

Family Disposable Income

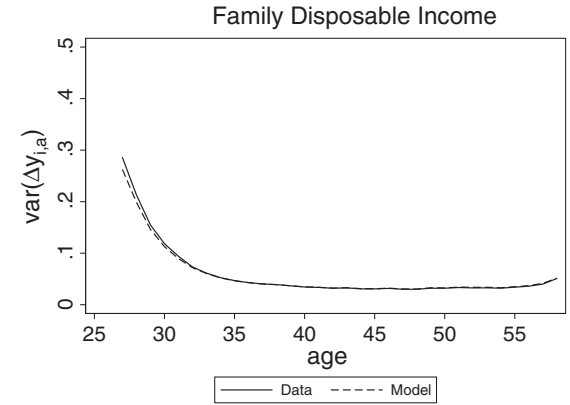

Fig. C.10. Variance profiles or residual income growth rates.

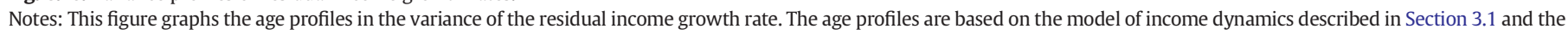

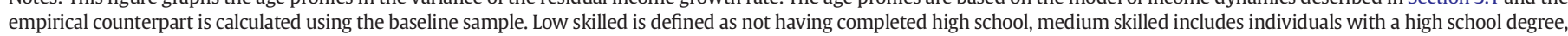
and high skilled consists of individuals who have attended college. The age profiles are adjusted for education-specific calendar time effects. 
a) Low-Skilled
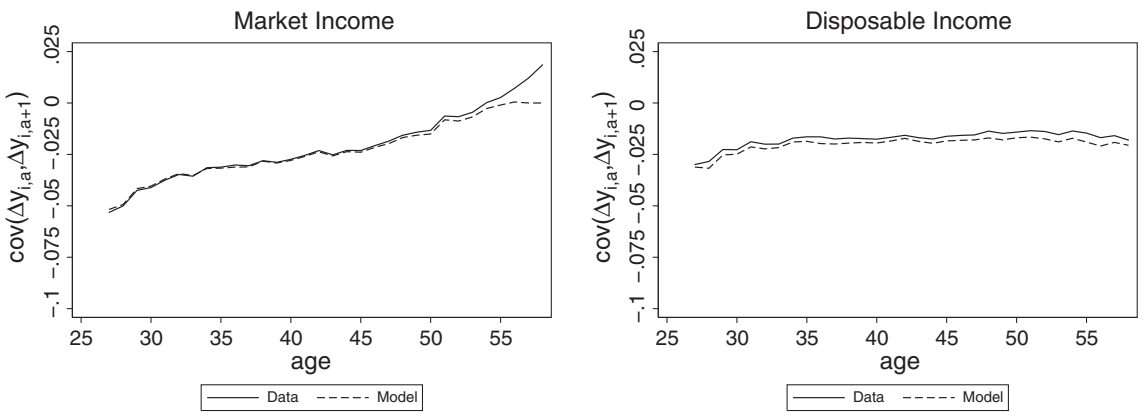

b) Medium-Skilled
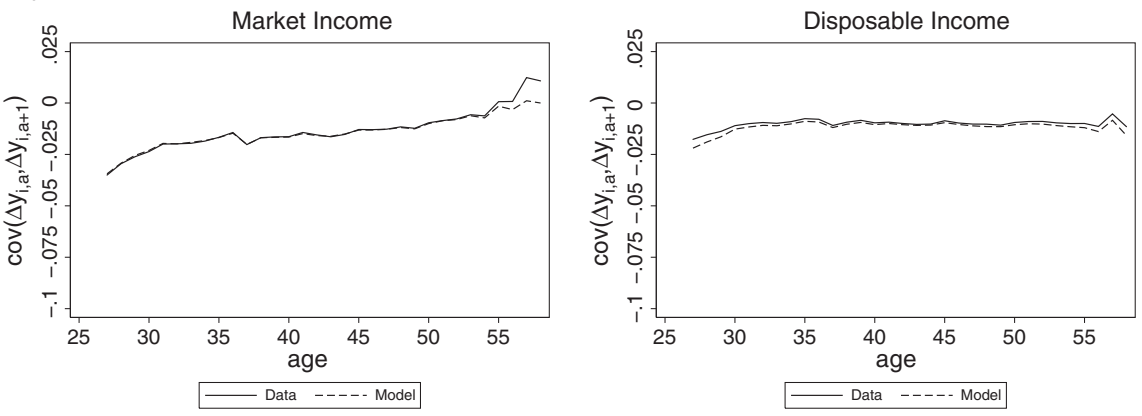

c) High-Skilled
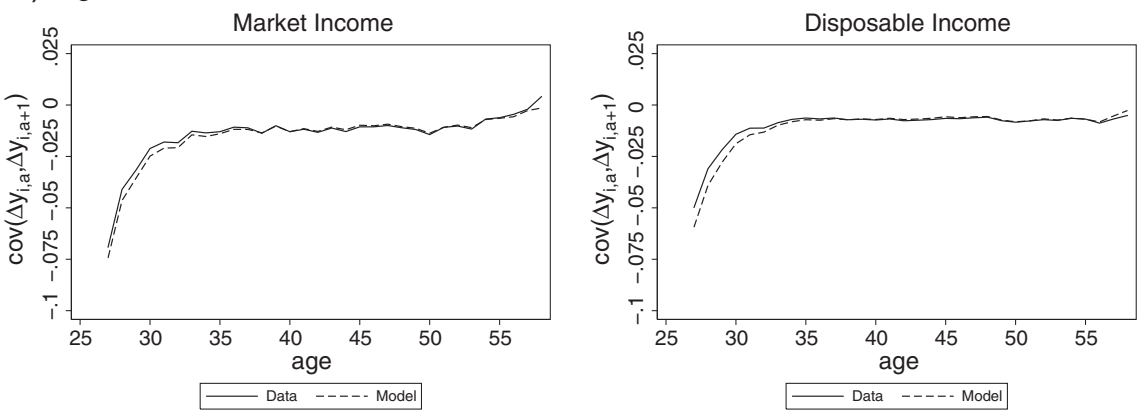
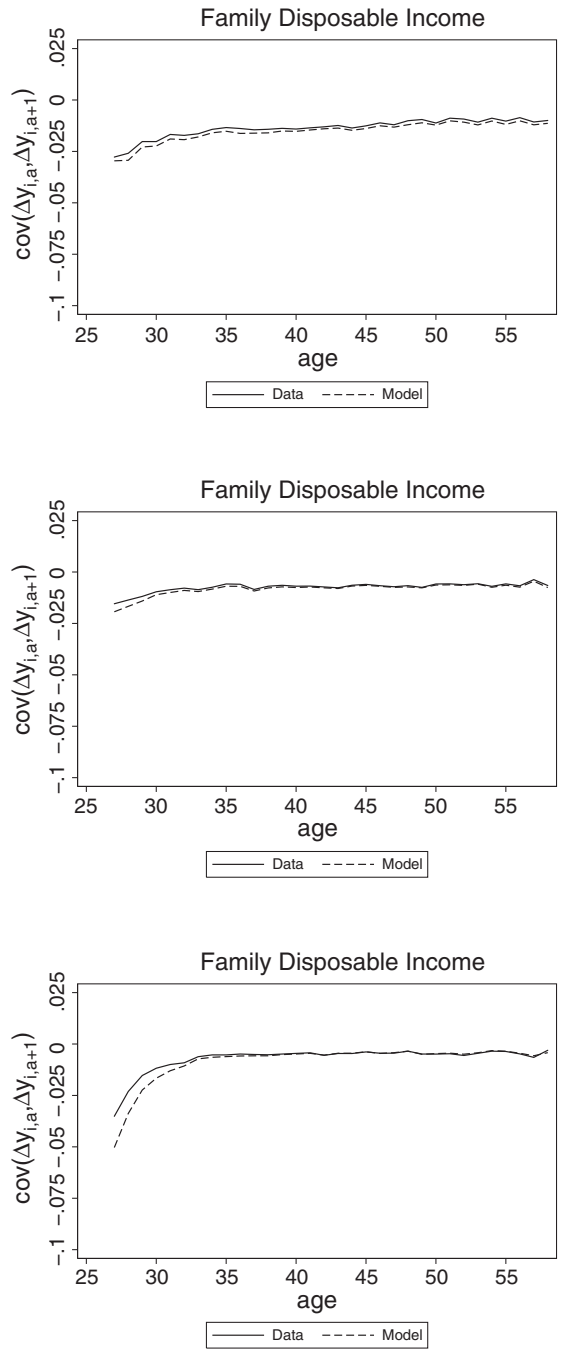

Fig. C.11. Covariance profiles of residual income growth rates

Notes: This figure graphs the age profiles in the covariance of the residual income growth rate at one lag. The age profiles are based on the model of income dynamics described in Section 3.1 and the empirical counterpart is calculated using the baseline sample. Low skilled is defined as not having completed high school, medium skilled includes individuals with a high school degree, and high skilled consists of individuals who have attended college. The age profiles are adjusted for education-specific calendar time effects.

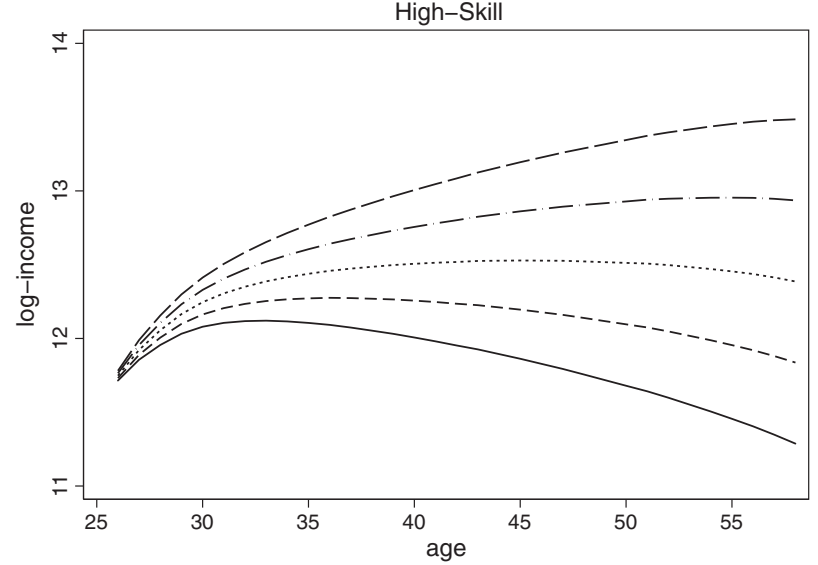

Fig. C.12. Heterogeneous income profiles in market income among the high-skilled. Notes: This figure graphs the heterogeneous profiles in individual market income for the high skilled. High skilled consists of individuals who have attended college. The dotted line in the middle shows the age profile for the average growth rate $(\beta=0)$ The lines above and below show age profiles for $\beta= \pm \sqrt{\operatorname{var}\left(\beta_{i}\right)}$ and $\beta= \pm 2 \times \sqrt{\operatorname{var}\left(\beta_{i}\right)}$. The profiles are based on the model of income dynamics described in Section 3.1 with $p_{a}=a-25$.

\section{References}

Atkinson, A., Rainwater, L., Smeeding, T., 1995. "Income Distribution in OECD Countries: Evidence from the Luxembourg Income Study," Report, OECD.

Baker, M., Solon, G., 2003. Earnings dynamics and inequality among Canadian men, 19761992: evidence from longitudinal income tax records. J. Labor Econ. 21, 267-288.

Blundell, R., Pistaferri, L., Preston, I., 2008. Consumption inequality and partial insurance. Am. Econ. Rev. 98, 1887-1921.

Blundell, R., Pistaferri, L., Saporta-Eksten, I., 2012. Consumption inequality and family labor supply. NBER Working paper. No. 18445, November.

DeBacker, J., Heim, B., Panousi, V., Ramnath, S., Vidangos, I., 2013. Rising inequality: transitory or persistent? New evidence from a panel of U.S. tax returns. Brookings Papers on Economic Activity, pp. 67-142.

Gourinchas, P.-O., Parker, J.A., 2002. Consumption over the life cycle. Econometrica 70, 47-89.

Guvenen, F., 2009. An empirical investigation of labor income processes. Rev. Econ. Dyn. $12,58-79$.

Haider, S.J., 2001. Earnings instability and earnings inequality of males in the United States: 1967-1991. J. Labor Econ. 19, 799-836.

Heathcote, J., Storesletten, K., Violante, G.L., 2005. Two views of inequality over the life cycle. J. Eur. Econ. Assoc. 3, 765-775.

Heckman, J.J., Lochner, L., Todd, P.E., 2003. Fifty years of mincer earnings regressions. Discussion papers. IZA.

Huggett, M., Ventura, G., Yaron, A., 2011. Sources of lifetime inequality. Am. Econ. Rev. 101, 2923-2954.

Kaplan, G., 2012. Inequality and the life cycle. Quant. Econ. 3, 471-525.

Karahan, F., Ozkan, S., 2013. On the persistence of income shocks over the life cycle: evidence, theory, and implications. Rev. Econ. Dyn. 16, 452-476. 
Kopczuk, W., Saez, E., Song, J., 2010. Earnings inequality and mobility in the United States: evidence from social security data since 1937. Q. J. Econ. 125, 91-128.

Lillard, L.A., Weiss, Y., 1979. Components of variation in panel earnings data: American scientists, 1960-1970. Econometrica 47, 437-454.

Lundberg, S., 1985. The added worker effect. J. Labor Econ. 3, 11-37.

MaCurdy, T.E., 1982. The use of time series processes to model the error structure of earnings in longitudinal data analysis. J. Econ. 18, 83-114.

Meghir, C., Pistaferri, L., 2011. Earnings, consumption and lifecycle choices. Handb. Labor Econ. 4, 773-854.

Mincer, J., 1974. Schooling, Experience, and Earnings. NBER Press.

Moffitt, R., Gottschalk, P., 2012. Trends in the transitory variance of male earnings: methods and evidence. J. Hum. Resour. 47 (1), 204-236.
Ostrovsky, Y., 2010. Long-run earnings inequality and earnings instability among Canadian men revisited, 1985-2005. B.E. J. Econ. Anal. Policy 10 (Article 20).

Ostrovsky, Y., 2012. The correlation of spouses' permanent and transitory earnings and family earnings inequality in Canada. Labour Econ. 19, 756-768.

Storesletten, K., Telmer, C.I., Yaron, A., 2004. Cyclical dynamics in idiosyncratic labor market risk. J. Polit. Econ. 112, 695-717.

Storesletten, K., Telmer, C.I. and Yaron, A., 2001. The welfare cost of business cycles revisited: finite lives and cyclical variation in idiosyncratic risk. Eur. Econ. Rev. 45, 1311-1339. 